\title{
TCAB1 prevents nucleolar accumulation of the telomerase RNA to
}

$$
\text { promote telomerase assembly }
$$

3

$$
\text { Han }{ }^{5} \text {, Kefei } \mathrm{Yu}^{5} \text {, Jens C. Schmidt }{ }^{1,6,{ }^{*}}
$$

$7{ }^{1}$ Institute for Quantitative Health Sciences and Engineering, Michigan State University,

${ }^{2}$ College of Osteopathic Medicine, Michigan State University, East Lansing, MI, U.S.A.

${ }^{3}$ Cellular and Molecular Biology Graduate Program, College of Natural Sciences,

$14{ }^{5}$ Department of Microbiology and Molecular Genetics, Michigan State University, East 


\section{Abstract}

21 Localization of a wide variety of RNAs to non-membrane bound cellular compartments

22 such as nucleoli, Cajal bodies, and stress-granules is critical for their function and

23 stability. The molecular mechanisms that underly the recruitment and exclusion of specific

24 RNAs from these phase-separated organelles is poorly understood. Telomerase is a 25 ribonucleoprotein (RNP), that is composed of the reverse transcriptase protein TERT, the

26 telomerase RNA (TR), and several auxiliary proteins that associate with TR, including

27 TCAB1. Here we show that, that in the absence of TCAB1, TR is trapped in the nucleolus,

28 while TERT localizes to the nucleoplasm and is excluded from the nucleolus, which

29 prevents telomerase assembly. Thus, nucleolar phase separation constitutes a barrier

30 that counteracts telomerase assembly and TCAB1 is required to exclude the telomerase

31 RNA from the nucleolus. Our work provides general insight into the mechanism and

32 functional consequences of RNA recruitment to non-membrane bound organelles and

33 proposes a new model explaining the critical role of TCAB1 in telomerase function. 


\section{Introduction}

35 Human cells contain a number of non-membrane bound organelles that carry out critical 36 cellular functions. For instance, nucleoli and Cajal bodies are phase-separated nuclear 37 organelles that play important roles in the biogenesis and maturation of many cellular

38 RNAs (Hyman et al., 2014; Mitrea and Kriwacki, 2016). Nucleoli and Cajal bodies contain

39 a wide range of small nucleolar and small Cajal body-specific RNAs (snoRNAs and 40 scaRNAs, respectively). A subset of these snoRNAs and scaRNAs are bound by the 41 H/ACA complex, which contains NOP10, NHP2, GAR1, and the pseudouridylase dyskerin, which modifies ribosomal and spliceosomal RNA precursors and other RNAs

43 (Angrisani et al., 2014). A key difference between snoRNAs and scaRNAs is the presence 44 of the Cajal-body box (CAB-box) motif in scaRNAs that directly associates with the 45 telomerase Cajal body protein 1 (TCAB1, also known as WRAP53) (Jády et al., 2004;

46 Schmidt and Cech, 2015; Venteicher et al., 2009). TCAB1 is required for the recruitment

47 of scaRNAs to Cajal bodies and in its the absence scaRNAs localize to the nucleolus

48 (Venteicher et al., 2009). Therefore, TCAB1 controls which phase-separated nuclear 49 organelle scaRNAs associate with. Importantly, the molecular mechanism by which

50 TCAB1 drives exclusion of scaRNAs from the nucleolus and facilitates their recruitment

51 to Cajal bodies is unknown. In addition, it is unclear whether miss-localization of scaRNAs 52 to the nucleolus has functional consequences.

54 The telomerase RNA (TR) is a scaRNA and like other scaRNAs its association with 55 nucleoli and Cajal bodies is controlled by TCAB1 (Schmidt and Cech, 2015). Telomere 56 maintenance by telomerase is essential for continuous proliferation of stem cell 
57 populations in the human body and most cancers require telomerase activity for their survival (Stewart and Weinberg, 2006). To compensate for the incomplete replication of chromosome ends, telomerase appends TTAGGG repeats to the telomeric single-

60 stranded overhang (Schmidt and Cech, 2015). Telomerase-mediated telomere

61 maintenance requires three critical steps: Telomerase assembly, telomerase recruitment

62 to telomeres, and telomeric repeat synthesis (Schmidt and Cech, 2015). Mutations in

63 several genes have been identified that cause deficiencies in one of these critical steps

64 and lead to a variety of diseases known as telomere syndromes, characterized by 65 premature depletion of stem cell populations (Armanios and Blackburn, 2012). In addition,

66 telomerase is inappropriately activated in $>85 \%$ of cancers (Stewart and Weinberg, 67 2006). While telomerase recruitment to telomeres (Nandakumar and Cech, 2013) and 68 telomerase catalysis (Wu et al., 2017) have been studied extensively, much less is known 69 about telomerase assembly. Importantly, telomerase assembly could be targeted to 70 reduce telomerase activity in cancer cells, or to increase telomerase function in patients 71 affected by genetically defined telomerase deficiency syndromes (Nagpal et al., 2020;

72 Shukla et al., 2020).

74 Telomerase is a complex ribonucleoprotein (RNP). The core components of telomerase 75 are the telomerase reverse transcriptase (TERT) protein, TR, the H/ACA complex, and 76 TCAB1 (Schmidt and Cech, 2015). The primary function of the H/ACA complex is to 77 stabilize TR, by directly binding to its 3'-end, preventing the exonucleolytic degradation of 78 TR (Stuart et al., 2015; Tummala et al., 2015). The 3'-end formation of TR is tightly 79 regulated by the competing activities of the poly-(A) polymerase PAPD5 and the nuclease 
80 PARN (Shukla et al., 2016; Tseng et al., 2015). Loss of TCAB1 function leads to telomere

81 attrition in a variety of cell lines (Chen et al., 2018; Venteicher et al., 2009; Vogan et al.,

82 2016; Zhong et al., 2011). In addition, multiple mutations in TCAB1 have been identified

83 that cause misfolding of TCAB1 and lead to dyskeratosis congenita, a telomere syndrome

84 (Freund et al., 2014; Zhong et al., 2011). While these observations highlight that TCAB1

85 is necessary for telomere maintenance, the underlying molecular mechanism is unclear.

86 Initially, it was proposed that TCAB1 is required for telomerase recruitment to telomeres

87 (Stern et al., 2012; Venteicher et al., 2009). A more recent study suggested that TCAB1

88 is required for the correct folding of $T R$, and that its absence causes a reduction in

89 telomerase activity (Chen et al., 2018). Importantly, all previous studies have come to the

90 conclusion that TCAB1 is not required for telomerase assembly.

92 Here we analyze telomerase assembly in intact cells and by purification of the telomerase

93 RNP and demonstrate that, contrary to previous findings, TCAB1 promotes telomerase

94 assembly in vivo. Using a combination of genetic perturbations, biochemical analysis of

95 telomerase function, cell biological approaches, and single-molecule live cell imaging, we

96 show that in the absence of TCAB1, TR is sequestered in the nucleolus while TERT is

97 excluded from the nucleolus. The spatial separation of TERT and TR that we observe in

98 our experiments is inconsistent with proper telomerase assembly. Furthermore, we show

99 that the limited amount of telomerase that can assemble in the absence of TCAB1 is fully

100 active and can localize to telomeres, suggesting that TCAB1 is not required for the

101 enzymatic function of telomerase or its recruitment to telomeres. In addition, we

102 demonstrate that the diffusion properties of TERT in cells that lack TCAB1 closely 
103 resemble those of TERT that is not bound to TR. We conclude that the sequestration of

104 TR in the nucleolus, when TCAB1 is absent, prevents its association with TERT, and that

105 this lack of telomerase assembly is the molecular mechanism underlying the critical role

106 of TCAB1 in telomere maintenance. Our results also demonstrate that the nucleolar

107 phase separation constitutes a barrier for telomerase assembly and suggest that

108 incompletely assembled RNPs are tightly associated with the nucleolus and do not readily

109 enter the nucleoplasm. 


\section{Results}

\section{Loss of TCAB1 leads to nucleolar accumulation of TR}

113 To confirm that TR is sequestered in the nucleolus in the absence of TCAB1, we

114 attempted to knock-out TCAB1 in parental HeLa cells and HeLa cells expressing

115 3xFLAG-HaloTag-TERT and mEos3.2-TRF2 from the endogenous TERT and TRF2 loci

116 (referred to as Halo-TERT from here on) using Cas9 and a single guide RNA targeting

117 the first exon of the TCAB1 locus (Figure 1 - figure supplement 1 ). We isolated clonal cell

118 lines that lacked a wild-type TCAB1 allele (Figure 1 - figure supplement 1) and analyzed

119 TCAB1 expression using Western blot. While a TCAB1 antibody targeting the N-terminus

120 of TCAB1 failed to detect TCAB1 in our knock-out clones (Figure 1 - figure supplement

121 1), antibodies raised against more C-terminal TCAB1 fragments detected a truncated

122 form of TCAB1 (Figure 1 - figure supplement 1). The size of the truncated TCAB1 is

123 consistent with the use of methionine 129 as an alternative start codon. Importantly, the

124 truncated form of TCAB1 localizes to Cajal bodies (Figure 1 - figure supplement 1) and

125 scaRNA localization is unaffected in its presence (Figure 1 - figure supplement 1),

126 indicating that it is at least partially functional. To avoid expression of the truncated TCAB1

127 variant, we knocked out TCAB1 using Cas9 with two guide RNAs to delete exons 2 and

1283 from the TCAB1 gene, which removes the coding sequence for residues 144-214 of

129 TCAB1 and results in a frame shift (Figure 1 - figure supplement 2). TCAB1 knock-out

130 was validated by Southern blot, PCR, Western blot, and immunofluorescence imaging

131 (IF, Fig. 1A-C, Figure 1 - figure supplement 2). Fluorescence in situ hybridization (FISH)

132 demonstrated that TR accumulates in the nucleolus in cells that lack TCAB1, as indicated

133 by co-localization of TR and nucleolar dyskerin signals. Importantly, expression of GFP- 
134 TCAB1 in TCAB1 knock-out cells rescued TR localization to Cajal bodies (Figure 1 -

135 figure supplement 2), confirming that the miss-localization of TR to nucleoli is caused by

136 absence of TCAB1. These observations confirm that TCAB1 is required to prevent TR

137 accumulation in the nucleolus (Fig. 1C).

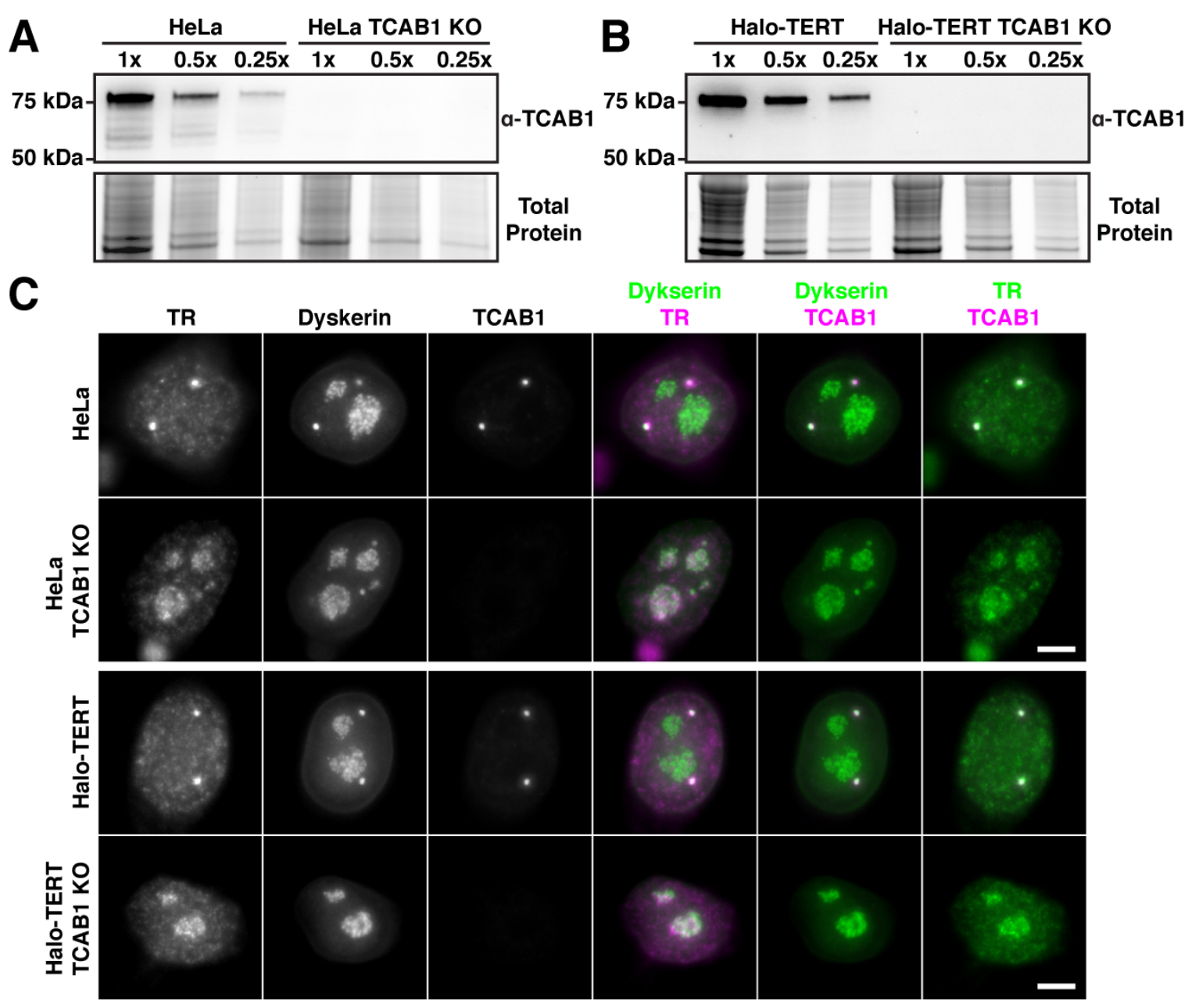

Figure 1. TR is localized to nucleoli in TCAB1 knock-out cells. (A-B) Western blot demonstrating the absence of TCAB1 protein in TCAB1 knock-out cell lines generated from (A) HeLa and (B) HaloTERT parental cell lines (probed with Proteintech TCAB1 antibody). (C) Immuno-fluorescence with anti-dyskerin and anti-TCAB1 antibodies coupled to fluorescence in-situ hybridization with probes against TR, demonstrating the absence of TCAB1 and TR localization to nucleoli in TCAB1 knock-out cells (scale bar $=5 \mu \mathrm{m})$.

\section{TERT is excluded from nucleoli}

140 Our previous observations demonstrated that TERT does not enter nucleoli in human

141 cancer cells (Schmidt et al., 2016). To confirm these results, we performed single- 
142 molecule imaging of 3xFLAG-HaloTag-TERT in living HeLa cells. Consistent with our

143 previous results, 3xFLAG-HaloTag-TERT was not observed entering or overlapping with

144 nucleoli, which are discernible as circular shapes in the nucleus under transmitted light

A

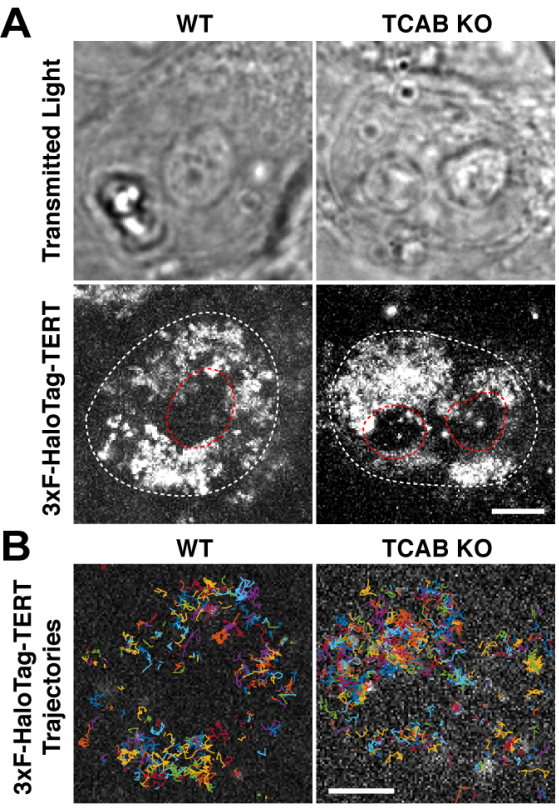

Figure 2. TERT is excluded from nucleoli in control and TCAB1 knock-out cells. (A) Maximum intensity projections of 2000 frames of 3xFLAG-HaloTag (JF646) TERT movies (bottom), demonstrating that the TERT signal does not overlap with the nucleolus detected as circular shape in the transmitted light image in control and TCAB1 knock-out cells (top, red dashed line, scale bar $=2 \mu \mathrm{m})$. (B) TERT particle trajectories from the cells shown in Fig. $2 \mathrm{~A}$, demonstrating that TERT molecules move parallel to or away from the nucleolus when located at the interface between the nucleolus and nucleoplasm.

illumination (Fig. 2A,B, Figure 2 - figure supplement 1, Movie 1,2). TERT trajectories in

147 close proximity to nucleoli revealed that their movement is biased away from the 148 nucleolus, suggesting that TERT is repelled by the nucleolus (Fig. 2B). To exclude the 149 possibility that nucleolar exclusion is a consequence of the 3xFLAG-HaloTag on the N150 terminus of TERT used in our experiments, we transiently expressed the 3xFLAG151 HaloTag fused to a nuclear localization sequence (NLS) in HeLa cells. Single-molecule 152 imaging demonstrated that the nuclear 3xFLAG-HaloTag signals overlapped with the 153 nucleolus (Figure 2 - figure supplement 1, Movie 3). Similar to the 3xFLAG-HaloTag 154 alone, 3xFLAG-HaloTag-dyskerin also localized to the nucleolus (Figure 2 - figure 155 supplement 1). These results demonstrate that 3xFLAG-HaloTag-TERT is excluded from 156 the nucleolus and that this exclusion is not caused by the 3xFLAG-HaloTag but instead 157 is an intrinsic property of the TERT protein. 
TERT and TR localize to distinct nuclear compartments in cells lacking TCAB1

160 It is well established that telomerase assembly is incomplete in human cancer cells, which

161 leads to substantial pools of TERT and TR that are not assembled into telomerase RNPS

162 (Xi and Cech, 2014). Together with our observation that TERT molecules rarely enter the

163 nucleolus (Schmidt et al., 2016) this suggests that both free TERT and assembled

164 telomerase RNPs do not enter the nucleolus. To test whether, like TR, TERT accumulates

165 in the nucleolus in cells lacking TCAB1, we carried out single-molecule imaging of

166 3xFLAG-HaloTag-TERT. Strikingly, TERT localization is unchanged in cells lacking

167 TCAB1 (Fig. 2A,B). This suggests that unlike TR, TERT does not accumulate in nucleoli

168 when TCAB1 is absent.

169 The experiments described so far demonstrate that TCAB1 is enriched in nucleoli

170 and TERT is excluded from nucleoli in cells lacking TCAB1, but they do not

171 simultaneously detect TERT and TR in the same cell. To overcome this limitation, we

172 over-expressed mCherry-TERT and TR in TCAB1 knock-out cells. In controls, TERT and

173 TR colocalized with dyskerin at telomeres marked by TRF2 (Fig. 3A). In addition, analysis

174 of the TERT signal across the nucleus and nucleolus revealed that TERT was depleted

175 from the nucleolus (Fig. 3B). Importantly, mCherry-dyskerin localized to the nucleolus,

176 demonstrating that the mCherry-tag does not lead to nucleolar exclusion of its fusion

177 partner (Figure 3 - figure supplement 1). In cells lacking TCAB1, mCherry-TERT was

178 diffusely localized in the nucleoplasm, localized to a subset of telomeres, and was

179 depleted from the nucleolus (Fig. 3A,C). Similar to endogenous TR, overexpressed TR 

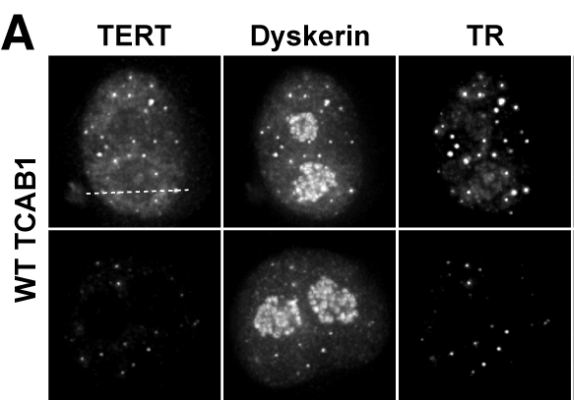

TRF2
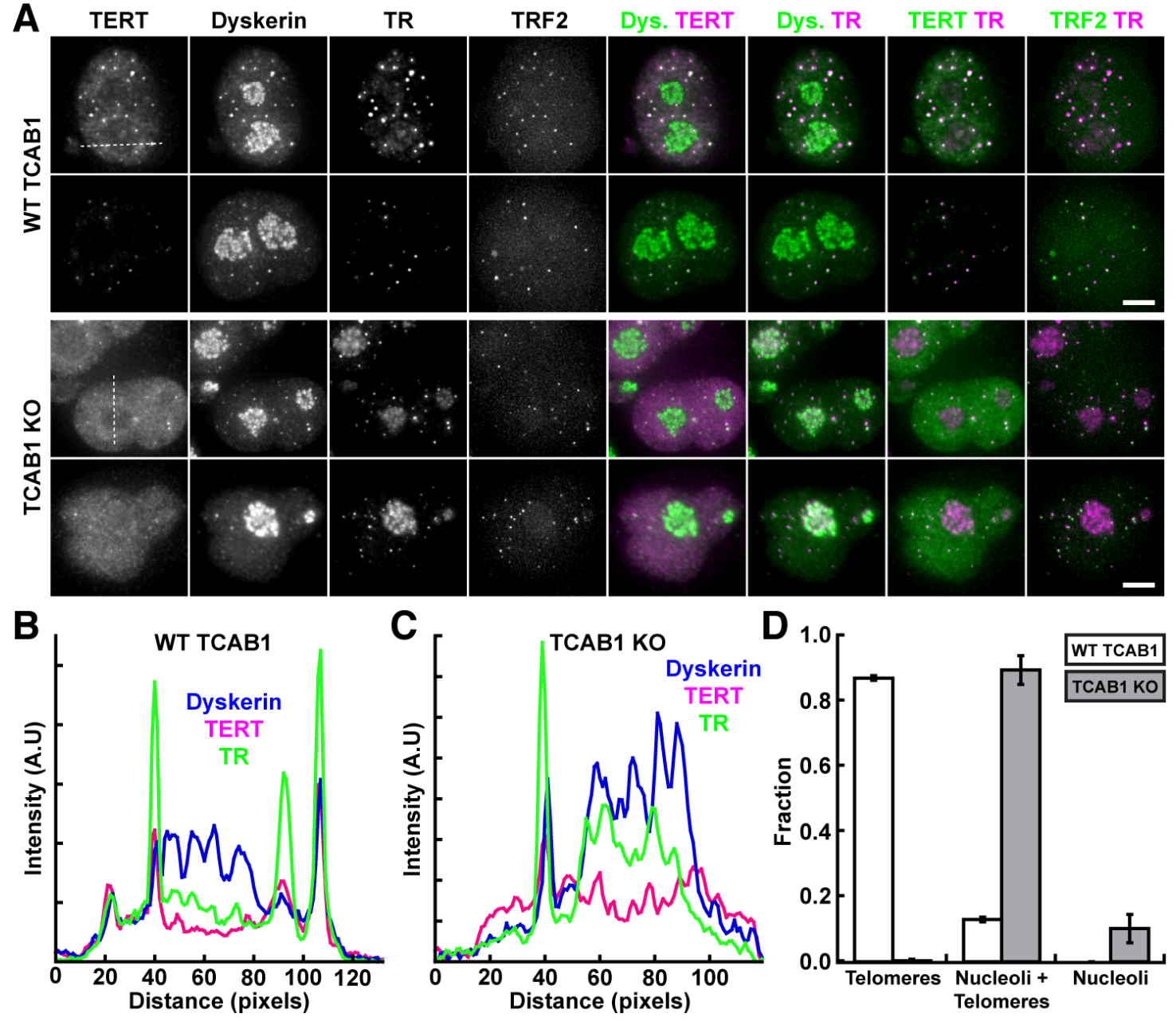

Figure 3. TERT and TR localize to distinct nuclear compartments in TCAB1 knock-out cells. (A) IF-FISH images of control and TCAB1 knock-out HeLa cells (3xFLAG-Halo-TERT, mEOS3.2-TRF2) overexpressing mCherry-TERT and TR (scale bar $=5 \mu \mathrm{m}$ ). Cells were probed with antibodies against dyskerin and mCherry, and FISH probes specific to TR. The intrinsic fluorescence of mEOS3.2-TRF2 was used to detect telomeres. TR and TERT are co-localized at telomeres in control cells. In TCAB1 knock-out cells, TR is enriched in nucleoli, while TERT is depleted from nucleoli in both control and TCAB1 knock-out cells. Both TERT and TR also localize to telomeres in TCAB1 knock-out cells. (B-C) Line scans of (B) control and (C) TCAB1 knock-out cells along the dashed white lines in Fig. 3A, demonstrating the enrichment of TR (green) in nucleoli (blue) in TCAB1 knock-out cells and the depletion of TERT (magenta) from nucleoli in both control and TCAB1 knock-out cells. (D) Quantification of the fraction of cells showing TR localization exclusively to telomeres, to telomeres and nucleoli, or only to nucleoli in cells over-expressing mCherry-TERT and TR ( 3 independent experiments, $>100$ cells per experiments, mean \pm standard deviation). 
185 untagged TERT and TR (Figure 3 - figure supplement 1) and untagged TERT was

186 excluded from nucleoli, confirming that nucleolar exclusion is an intrinsic property of

187 TERT (Figure 3 - figure supplement 1). Together these results demonstrate that, when 188 overexpressed in TCAB1 knock-out cells, TR is enriched in nucleoli and TERT is depleted

189 from nucleoli, consistent with a failure of nucleolar TR to assemble with TERT. In addition,

190 our observations suggest that overexpression can partially overcome the sequestration

191 of TR in the nucleolus when TCAB1 is absent, allowing a fraction of TR to bind to TERT

192 and localize to telomeres.

\section{TCAB1 promotes telomerase RNP assembly}

195 Previous studies by other laboratories have concluded that telomerase assembly is 196 unaffected by the absence of TCAB1 and whether TCAB1 is required for telomerase 197 activity is controversial (Chen et al., 2018; Venteicher et al., 2009; Vogan et al., 2016).

198 To assess the role of TCAB1 in telomerase assembly, we overexpressed TERT and TR 199 in parental HeLa and TCAB1 knock-out cells and immuno-purified TERT using a well200 established anti-TERT antibody (Cohen et al., 2007). To quantify telomerase assembly,

201 we measured TERT levels by Western blot and determined the amount of TR co-purified 202 using Northern blot (Fig. 4A,B). The amount of TR associated with TERT purified from 203 TCAB1 knock-out cells was significantly reduced compared to parental cells, for both 204 HeLa $(31 \pm 9 \%$, mean \pm SEM, $p=0.001)$ and Halo-TERT $(33 \pm 4 \%$, mean \pm SEM, $p=$ 205 0.001) cell lines (Fig. 4D). As a second measure of telomerase RNP assembly we 206 quantified the amount of dyskerin associated with TERT (Fig. 4C). Since TR bridges 207 TERT and dyskerin, dyskerin co-purified with TERT directly reports on the presence of 
210 controls for both HeLa $(22 \pm 8 \%$, mean \pm SEM, $p=0.002)$ and Halo-TERT $(32 \pm 9 \%$,

211 mean \pm SEM, $p=0.004$ ) cell lines (Fig. 4C,E). Importantly, we also confirmed that TCAB1

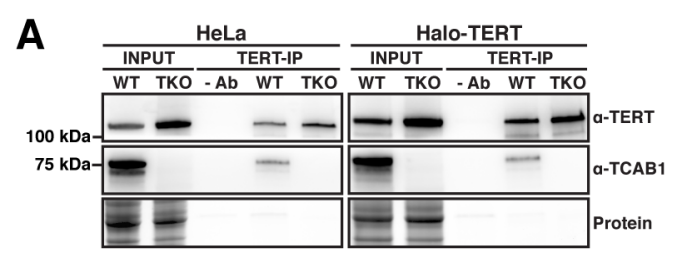

B
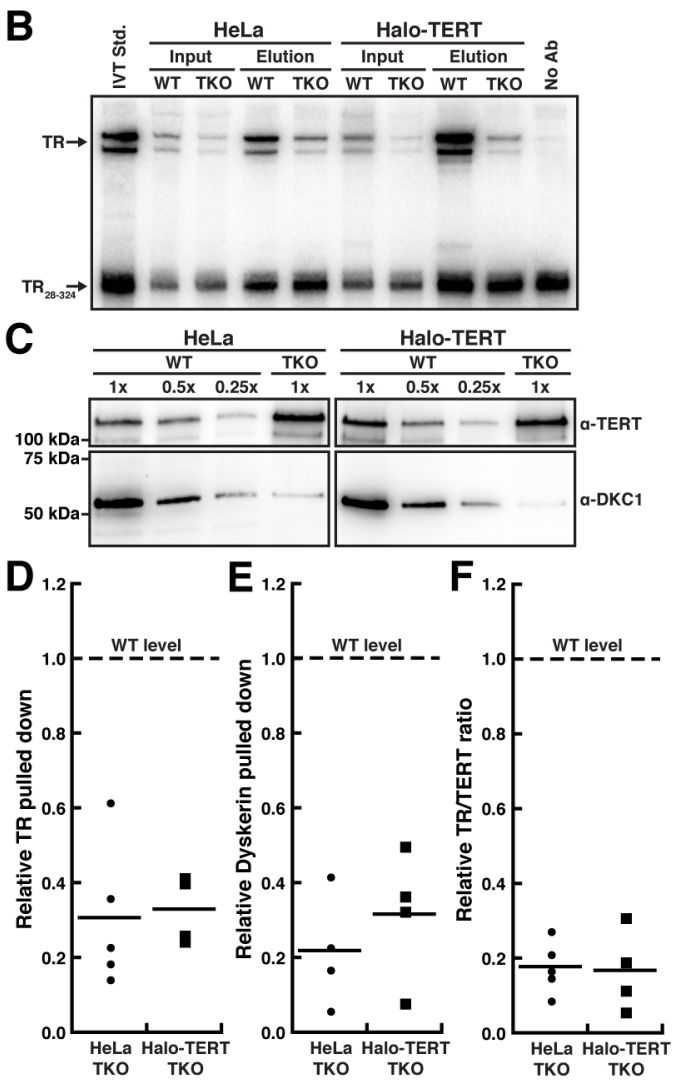

Figure 4. Telomerase Assembly is reduced in the absence of TCAB1. (A) Western blots analyzing TERT immuno-purification (using a sheep anti-TERT antibody) probed with a rabbit anti-TERT antibody (Abcam) and a TCAB1 antibody. (B) Northern blot of RNA extracted from input and purified TERT samples probed with three radiolabeled DNA oligonucleotides complementary to TR. Standards are in vitro transcribed full-length TR and truncated $\mathrm{TR}_{28-324}$. $\mathrm{TR}_{28-324}$ was added to samples prior to RNA extraction as loading and recovery control. (C) Western blots to analyze immuno-purified telomerase RNP composition. A single membrane was cut into two pieces that were probed with TERT and dyskerin antibodies, respectively. (D-F) Quantification of the amount of (D) TR, (E) dyskerin, and (F) the ratio of TR to TERT in TERT purifications from TCAB1 knock-out cells compared to parental controls ( $n=4-5$, mean). The dashed lines indicate the level in telomerase purified from wild-type TCAB1 control cells which was normalized to 1.0 .

214 measure of telomerase assembly, we determined the ratio between TR and TERT (Fig.

$2154 A, B, F)$, which was also significantly reduced when TERT was purified from TCAB1

216 knock-out cells (HeLa: $18 \pm 3 \%$, mean \pm SEM, $p<0.001$, Halo-TERT: $17 \pm 5 \%$, mean \pm

217 SEM, $p<0.001)$. Altogether, these results demonstrate that telomerase assembly is 
218 significantly reduced in the absence of TCAB1 and that overexpression of TERT and TR

219 is not sufficient to overcome this defect in telomerase RNP formation.

\section{TCAB1 is not required for telomerase catalytic activity}

222 Previous work by others has suggested that the defects in telomere maintenance

A TERT-IP from HeLa Cells

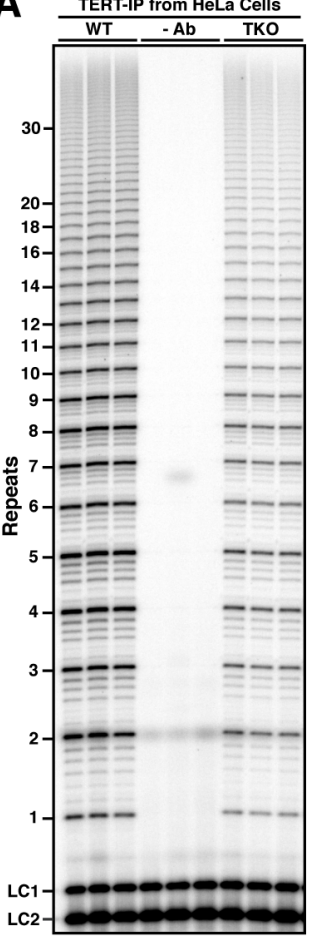

$3 \frac{\text { TERT-IP from Halo-TERT Cells }}{\text { WT }- \text { Ab TKO }}$

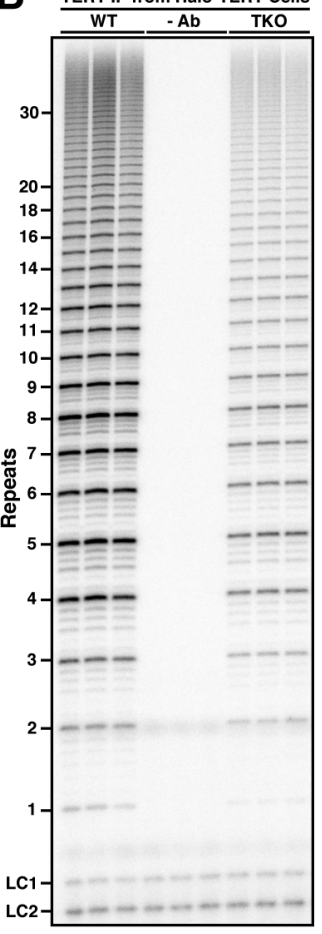

C

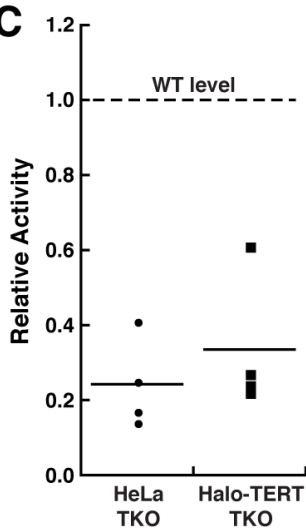

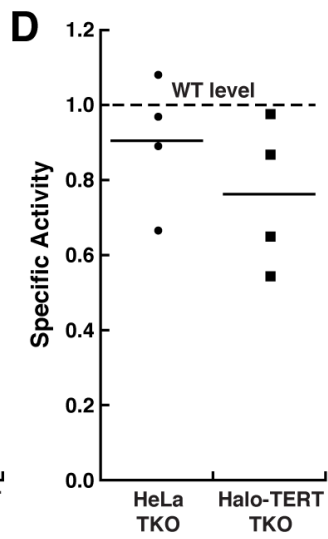

Figure 5 . The specific activity of telomerase is unchanged in the absence of TCAB1. (A-B) Direct telomerase extension assay of telomerase immunopurified from parental (WT) and TCAB1 knock-out (TKO) (A) HeLa and (B) Halo-TERT cell lines. LC1 and LC2, radiolabeled DNA oligonucleotide loading controls. In Ab samples the TERT antibody was omitted during the immuno-purification. (C) Quantification of telomerase activity in samples from TCAB1 knock-out cells relative to parental controls ( $n=4$, mean). (D) Specific activity of telomerase purified from TCAB1 knock-out cells relative to parental controls ( $n=4$, mean). Specific activity was calculated by dividing the relative activity (see Fig. $5 \mathrm{C}$ ) by the relative amount of TR present in immuno-purified TERT samples (see Fig. 4D). The dashed lines indicate the activity level in telomerase purified from wild-type TCAB1 control cells which was normalized to 1.0. 
224 observed in TCAB1 knock-out cells are the consequence of reduced telomerase activity

225 caused by the miss-folding of the telomerase RNA rather than impaired telomerase

226 assembly (Chen et al., 2018). This TR folding model predicts that the specific activity of

227 telomerase is reduced. To determine the specific activity of telomerase purified from

228 TCAB1 knock-out cells, we measured telomerase activity using the direct telomerase

229 extension assays (Fig. 5A,B, Figure 5 - figure supplement 1), and divided the activity by

230 the amount of TR in the respective telomerase sample (Fig. 4B,D), which is a direct

231 measure of the quantity of telomerase RNP present. Total activity of telomerase purified

232 from TCAB1 knock-out cells was significantly reduced relative to telomerase purified from

233 parental controls telomerase (Fig. 5C, HeLa: $24 \pm 6 \%$, mean \pm SEM, $p<0.001$, Halo-

234 TERT: $34 \pm 9 \%$, mean \pm SEM, $p=0.005)$, consistent with observations by others (Chen

235 et al., 2018). In contrast, the specific activity of telomerase derived from cells that lack

236 TCAB1 was not significantly reduced compared to controls (Fig. 5D, HeLa: $90 \pm 9 \%$, mean

$237 \pm$ SEM, $p=0.35$, Halo-TERT: $76 \pm 10 \%$, mean \pm SEM, $p=0.1)$. Together these

238 observations suggest that TCAB1 is required for telomerase assembly and that the limited

239 amount of telomerase RNP that can form in the absence of TCAB1 is fully active.

241 TCAB1 is required for telomerase assembly in living cells

242 The experiments presented thus far demonstrate that telomerase assembly is 243 significantly reduced in the absence of TCAB1 but were carried out in fixed cells or cell 244 lysates. To analyze telomerase assembly in intact cells, we carried out live cell single245 molecule imaging of 3xFLAG-HaloTag-TERT and determined the diffusion coefficient of 246 TERT particles (Fig. 6A, Movie 4, Figure 6 - figure supplement 1). The diffusion co- 
247 efficient is a measure of the rate of movement of a molecule and depends on the size of

248 the complex it is part of and reports on molecular interactions formed with other sub-

249 cellular structures. Analysis of the diffusion coefficients of TERT trajectories in control

250 cells revealed three distinct populations of TERT particles (Fig. 6B-D). A static population

$251\left(D_{s}=0.03 \mu m^{2} / \mathrm{s}, 12 \%\right)$ which likely represents assembled telomerase RNPs bound to

252 telomeres or Cajal bodies, a slowly diffusion population $\left(D_{\mathrm{F} 1}=0.35 \mu \mathrm{m}^{2} / \mathrm{s}, 28 \%\right.$ ) and a

253 rapidly diffusing population $\left(\mathrm{D}_{\mathrm{F} 2}=1.54 \mu \mathrm{m}^{2} / \mathrm{s}, 60 \%\right)$. The slowly diffusing population likely

254 includes assembled telomerase RNPs, while the rapidly diffusing particles represents

255 TERT molecules, which are not assembled with TR (Fig. 6B). Importantly, these diffusion

256 coefficients are largely consistent with our previous results using a distinct method to

257 determine their values (Schmidt et al., 2016). In the absence of TCAB1, the diffusion

258 coefficient of the freely diffusing TERT population was increased $\left(D_{\mathrm{F} 2}=1.78 \pm 0.04 \mu \mathrm{m}^{2} / \mathrm{s}\right.$,

259 mean \pm SEM, $p=0.04$, Fig. 6 C) and the fraction of the TERT populations that includes

260 assembled telomerase RNPs was significantly reduced $\left(F_{\text {Slow }+ \text { Static }}=29 \pm 1 \%\right.$, mean \pm

261 SEM, $p=0.02$, Fig. 6D). This observation is consistent with our model that in the absence

262 of TCAB1, telomerase assembly is defective. To determine the degree to which

263 telomerase assembly is affected in cells lacking TCAB1, we knocked out TR, completely

264 abolishing telomerase assembly. TR knock-out was confirmed by PCR and Sanger

265 sequencing, FISH, and qPCR (Figure 6 - figure supplement 1 ). Similar to control cells

266 and TCAB1 knock-out cells, TERT was also excluded from nucleoli in cells lacking TR

267 (Fig. S6D). Strikingly, the diffusion coefficients and the fraction of slow and static TERT

268 particles in cells lacking TR closely resembled those of TCAB1 knockout cells (Fig. 6A-

269 D, Figure 6 - figure supplement 1, Movie 4-5). To analyze the 


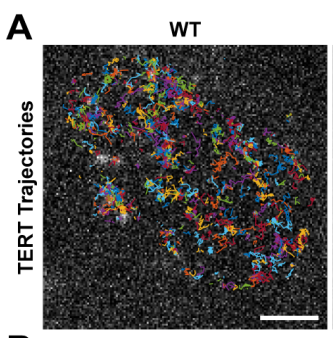

B

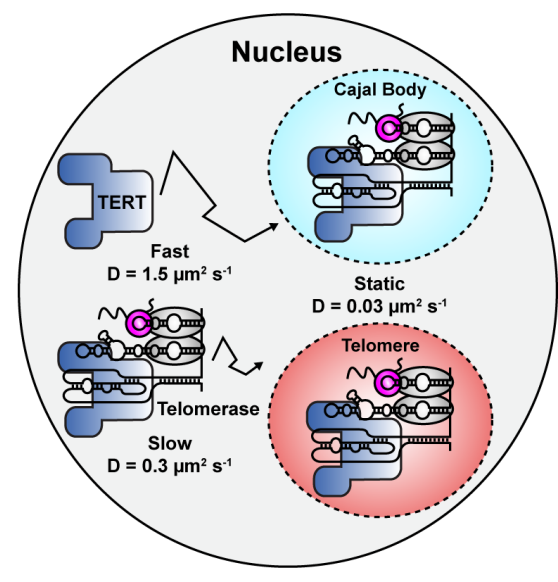

E

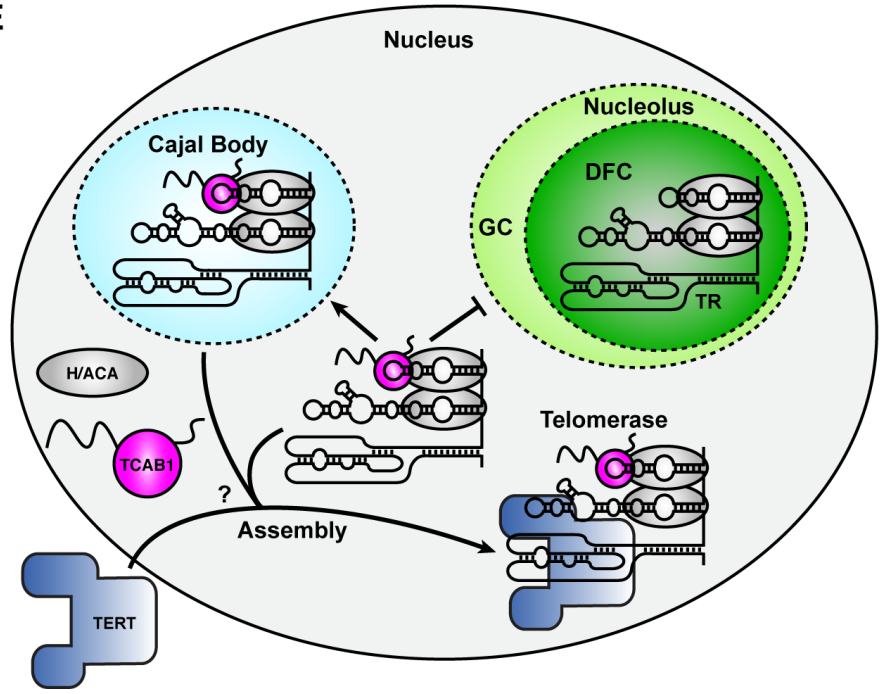

Figure 6. Telomerase assembly is reduced in TCAB1 knock-out cells. (A) TERT particle trajectories from control, TCAB1 knock-out, and TR knock-out cells expressing 3xFLAG-HaloTag TERT (JF646, scale bar $=2 \mu \mathrm{m}$ ). (B) Diagram of distinct populations of TERT particles detected in control cells. (C) Diffusion coefficient of the rapidly diffusing TERT population in control, TCAB1 knockout, and TR knock-out cells ( 3 independent experiments, $>15$ cells per experiment per cell line, mean \pm standard deviation, complete data in Fig. S6D). (D) Fraction of slow plus static TERT particles in control, TCAB1 knock-out, and TR knock-out cells expressing 3xFLAG-HaloTag TERT (3 independent experiments, $>15$ cells per experiment per cell line, mean \pm standard deviation, complete data in Fig. S6D). (D) Model for the regulation of telomerase assembly by TCAB1. In the absence of TCAB1, TR is sequestered in the dense fibrillar component (DFC) of the nucleolus, which is separated from the nucleoplasm by the granular component (GC) of the nucleolus. 
271 interaction of TERT with telomeres, we filtered out TERT trajectories that came into

272 proximity with telomeres marked by mEOS3.2-TRF2, as previously described (Schmidt

273 et al., 2016). To assess the interaction of TERT with telomeres, we plotted the step-size

274 vs. the distance from the closest telomere for each step of these trajectories (Figure 6 -

275 figure supplement 2). In control cells, we observed an enrichment of smaller step sizes

276 and particles in close proximity to telomeres, consistent with TERT interactions with the

277 telomere (Figure 6 - figure supplement 2). In contrast, TERT trajectories from TCAB1

278 knock-out cells lacked this enrichment, and the step size vs. distance from the closest

279 telomere plots were identical to those from TR knock-out cells (Figure 6 - figure

280 supplement 2). In addition, diffusion analysis using SpotOn revealed that the fraction of

281 static TERT particles at telomeres was reduced from $12 \%$ in control cells to $4-5 \%$ in

282 TCAB1 and TR knock-out cells (Figure 6 - figure supplement 2). These observations

283 indicate that in the absence of either TCAB1 or TR, stable interactions of telomerase with

284 telomeres occur at a lower frequency because they require base pairing of TR with the

285 chromosome end (Schmidt et al., 2018). Together these results demonstrate that

286 telomerase assembly is strongly reduced in the absence of TCAB1. 


\section{Discussion}

290 The experiments described in this study demonstrate that TCAB1 promotes telomerase

291 assembly. In the absence of TCAB1 the telomerase RNA is targeted to the nucleolus via

292 its association with dyskerin and other components of the H/ACA complex. In contrast to

293 TR, TERT cannot enter the nucleolus, preventing its association with TR in cells that lack

294 TCAB1. This demonstrates that nucleolar phase separation counteracts telomerase

295 assembly. In addition, we demonstrate that sequestration of TR in the phase-separated

296 nucleolus in the absence of TCAB1 can be partially overcome by telomerase

297 overexpression, leading to telomerase assembly and localization to telomeres. This

298 suggests that the nucleolus has a limited capacity to accommodate cellular RNAs and

299 that TCAB1 is not required for telomerase recruitment to telomeres. Finally, we

300 demonstrate that while telomerase assembly is limited the specific activity of telomerase

301 is unchanged in the absence of TCAB1, which excludes a role of TCAB1 in telomerase

302 catalytic function. Altogether our work completely reshapes our understanding of the role

303 of TCAB1 in telomerase function in human cells and provides insight into the role phase-

304 separated organelles play in RNP assembly and function.

306 TCAB1 promotes telomerase assembly

307 The importance of TCAB1 for telomere maintenance is undisputed (Chen et al., 2018;

308 Venteicher et al., 2009). Knock-out or depletion of TCAB1 results in telomere shortening

309 (Chen et al., 2018; Venteicher et al., 2009; Vogan et al., 2016). All previous work also

310 concluded that TR is enriched in the nucleolus in the absence of TCAB1 (Chen et al.,

311 2018; Stern et al., 2012; Venteicher et al., 2009; Vogan et al., 2016; Zhong et al., 2011). 
312 Finally, all prior studies propose that TCAB1 is not required for telomerase assembly but

313 instead plays a role telomerase trafficking to Cajal bodies and telomeres or is required for

314 telomerase catalysis (Chen et al., 2018; Stern et al., 2012; Venteicher et al., 2009; Vogan

315 et al., 2016; Zhong et al., 2011). In contrast, our results demonstrate that in the absence

316 of TCAB1, TERT and TR are localized to the distinct sub-cellular compartments, the

317 nucleoplasm and the nucleolus, respectively. This spatial separation strongly reduces

318 telomerase assembly, which leads to reduced number of telomerase RNPs per cell and

319 in turn telomere shortening. Our conclusions are based on a variety of quantitative

320 approaches analyzing telomerase assembly by purification of the telomerase RNP and in

321 intact cells. Analysis of TERT localization in living and fixed cells clearly demonstrates

322 that TERT is excluded from the nucleolus. Importantly, we confirmed that the nucleolar

323 exclusion of TERT is not a consequence of the protein-tags used to visualize TERT in

324 these experiments. Purification of telomerase from TCAB1 knock-out cells further

325 confirmed that TCAB1 is required for telomerase assembly in vivo. The amount of TR and

326 dyskerin associated with TERT is substantially reduced in the absence of TCAB1. Finally,

327 we used live cell single-molecule imaging to demonstrate that the diffusion pattern of

328 TERT molecules and their interactions with telomeres are significantly different in TCAB1

329 knock-out cells compared to control cells. More importantly, TERT dynamics in the

330 absence of TCAB1 are comparable to those in TR knock-out cells where telomerase

331 assembly is impossible. Altogether, these observations support our model that TCAB1

332 promotes telomerase assembly by extracting TR from the nucleolus to facilitate its

333 assembly with TERT. Our model is further supported by work from Vogan et al., which

334 demonstrated that truncated TR (hTRmin) that lacks the H/ACA region and therefore 
335 cannot bind dyskerin, accumulates in the nucleoplasm and is excluded from nucleoli

336 (Vogan et al., 2016). Importantly, in cells that express hTRmin, TCAB1 is not required for

337 telomere maintenance (Vogan et al., 2016), consistent with TCAB1 promoting telomerase

338 assembly by extracting TR from the nucleolus.

340 TCAB1 is not required for telomerase catalysis

341 Previous work by others has reported conflicting results regarding the role of TCAB1 in

342 telomerase catalysis, ranging from full enzymatic activity in initial reports to substantial 343 activity defects in the most recent study (Chen et al., 2018; Venteicher et al., 2009; Vogan

344 et al., 2016). Importantly, both our work and the only other study that analyzed the role of

345 TCAB1 in telomerase activity using the "gold-standard" direct telomerase extension assay

346 concluded that telomerase activity is reduced to approximately $20-30 \%$ in the absence of

347 TCAB1. In both cases, telomerase was purified from TCAB1 knock-out cells using an 348 antibody raised against TERT prior to measuring telomerase activity. While both studies 349 concur on the degree to which telomerase activity is reduced in the absence of TCAB1, 350 the proposed underlying molecular mechanisms differ. Chen et al. propose that TCAB1

351 is required for proper folding of the CR4/CR5 region of the telomerase RNA, which directly 352 associates with TERT, without affecting telomerase assembly (Chen et al., 2018). Recent 353 structural analysis of the telomerase RNP from human cells revealed that TCAB1 is 354 located far away from the CR4/CR5 region of TR (Figure 6 - figure supplement 3)(Ghanim 355 et al., 2021). TCAB1 resides within the H/ACA lobe of while CR4/CR5 is located within 356 the catalytic lobe of the telomerase RNP. The two lobes are connected by the P1 and 357 P4.2 helices which can adopt multiple conformations. Importantly, TCAB1 was not located 
358 near the CR4/CR5 region of TR in any of the observed telomerase RNP conformations

359 (Ghanim et al., 2021). Although it is possible that telomerase can adopt additional

360 conformations, based on the currently available structural information it is difficult to

361 rationalize a molecular mechanism by which TCAB1 could specifically promote CR4/CR5

362 folding. In addition, due to the miss-folding of TR telomerase was proposed to adopt a

363 low activity state in the absence of TCAB1. Experimentally such a low activity state would

364 manifest itself as a reduction in the specific activity of telomerase (telomerase activity per

365 assembled telomerase RNP). Our experiments strongly suggest that, while telomerase

366 assembly is reduced in the absence of TCAB1, the limited amount of telomerase that can

367 assemble is fully active (i.e. does not have reduced specific activity). Both, our enzymatic

368 analysis and the positioning of TCAB1 within the telomerase RNP do not support a role

369 of TCAB1 in TR folding and telomerase catalysis but are fully consistent with TCAB1

370 promoting telomerase assembly.

372 TCAB1 is not necessary for telomerase recruitment to telomeres

373 TCAB1 is necessary for the localization of scaRNAs to Cajal bodies and previous work

374 suggested that it is also required for telomerase recruitment to telomeres (Stern et al.,

375 2012; Venteicher et al., 2009). Our observations demonstrate that when TERT and TR

376 are overexpressed in TCAB1 knockout cells a fraction of TERT can assemble with TR an

377 localize to telomeres. Telomerase recruitment to telomeres requires a direct interaction

378 between TERT and TPP1 (Nandakumar et al., 2012; Schmidt et al., 2014; Zhong et al.,

379 2012), therefore TR that localizes to telomeres must be assembled with TERT. These

380 results are fully consistent with our model that the spatial separation of TR and TERT in 
381 the absence of TCAB1 prevents telomerase assembly. When TR is overexpressed the

382 capacity of the nucleolus to sequester TR may be saturated and excess TR can assemble

383 with TERT and localize to telomeres. Importantly, our observations also suggests that

384 TCAB1 is not required for telomerase localization to telomeres.

386 Molecular mechanism of telomerase RNP assembly

387 The results presented in this study demonstrate that, in the absence of TCAB1, 388 telomerase assembly is reduced but not completely abolished. It is likely that TR bound 389 by the H/ACA complex is in an equilibrium between localizing to nucleoplasm and the 390 nucleolus. In the presence of TCAB1, this equilibrium is shifted towards the nucleoplasm 391 allowing TR to associate with TERT (Fig. 6E). In contrast, in the absence of TCAB1, the 392 majority of TR is trapped in the nucleolus effectively reducing the amount of TR that is 393 available for assembly with TERT. In addition, the nucleolus disassembles during mitosis 394 and does not reform until early G1-phase of the cell cycle (Hernandez-Verdun, 2014). In 395 cells lacking TCAB1, nucleolar disassembly should lead to the release of TR, which could 396 transiently allow telomerase assembly during cell division or prior to reformation of the 397 nucleolus in G1-phase. Both, a reduction of TR available for assembly and transient 398 assembly during cell division are plausible models to explain the limited amount of 399 telomerase that can form in the absence of TCAB1.

$400 \quad$ The sub-cellular location and order in which telomerase RNP components 401 associate with TR in human cells is largely unknown (Fig. 6E). Our results demonstrate 402 that telomerase does not assemble in the nucleolus. In addition, it is unlikely that 403 telomerase assembly requires intact Cajal bodies. Multiple studies have shown that 
404 knock-out of coilin, which eliminates Cajal bodies, does not impact telomerase activity or 405 telomere maintenance (Chen et al., 2018; Vogan et al., 2016). Our single-molecule live 406 cell imaging of TERT has demonstrated that TERT is almost exclusively localized to the 407 nucleus, it is therefore likely that human telomerase assembles in the nucleoplasm. But 408 it cannot be ruled out that in human cells TR is transiently exported to the cytoplasm to 409 assemble with nascent TERT protein, as is the case in S. cerevisiae (Gallardo et al., 410 2008).

411 The H/ACA complex has been shown to associate with scaRNAs at the site of their 412 transcription (Darzacq et al., 2006). While co-transcriptional association of the H/ACA 413 complex with TR has not been demonstrated, binding of the H/ACA complex proteins to 414 TR is likely the first step in telomerase RNP assembly, because of their critical role in TR 415 stability and 3'-end formation (Stuart et al., 2015; Tummala et al., 2015). Once TR is 416 bound to dyskerin, TR will be targeted to the nucleolus, unless it associates with TCAB1 417 (Fig. 6E). Like TERT, TCAB1 is excluded from nucleoli (Fig. 1, Fig. S2D), and dyskerin 418 an TR are localized to the dense fibrillar component (DFC, Fig. 6E) of the nucleolus (Yao 419 et al., 2019), which is separated from the nucleoplasm by the granular component (GC, 420 Fig. 6E) (Lafontaine et al., 2021). Thus, it is unlikely that TCAB1 can bind to TR localized 421 to the DFC of the nucleolus. We therefore believe that TCAB1 association with TR 422 prevents the entry of TR into the nucleolus rather than extracting TR which is already 423 localized to the DFC of the nucleolus. The association of TR with either TERT or TCAB1 424 should prevent its sequestration in the nucleolus, but the order in which TERT and TCAB1 425 bind to TR cannot be inferred from our experiments, or observation by others. Since 
426 TCAB1 is substantially more abundant than TERT it is likely that TCAB1 encounters TR

427 before TERT does.

\section{Regulation of RNP assembly by nucleolar phase-separation}

430 In addition to the mechanistic insight into the role of TCAB1 in telomerase function, our 431 results also demonstrate that nucleolar phase separation can effectively regulate

432 telomerase RNP assembly in the nucleus of human cells. How RNA molecules are 433 specifically recruited into, excluded from, or expelled from non-membrane bound 434 organelles is a key unanswered question. One model suggests that gradual replacement 435 of non-specific, multivalent interactions of pre-ribosomal RNAs with nucleolar proteins 436 such as NPM1 and fibrillarin, with specific, high-affinity interactions with ribosomal 437 proteins leads to the ejection of mature ribosomal subunits from the nucleolus (Riback et 438 al., 2020). In this model a key driving force for the retention of RNA in the nucleolus is 439 regions of RNA not yet bound by ribosomal proteins, that are available to interact with 440 nucleolar proteins (Riback et al., 2020). By analogy, this model would explain why TR 441 bound by the H/ACA complex but not TERT would be sequestered in the nucleolus. In 442 addition to the interactions formed by the H/ACA complex with nucleolar proteins and 443 RNA, the regions of TR that are bound by TERT in the context of telomerase (i.e. the 444 pseudoknot, template, and CR4/CR5) would be available to form non-specific, multivalent 445 interactions with nucleolar proteins to strengthen the association of TR with the nucleolus 446 and prevent its release.

447 TR is a unique among the scaRNAs because it contains the additional domains 448 that associate with TERT. Most other box H/ACA scaRNAs are substantially shorter $(<150$ 
449 nucleotides) than TR (451 nucleotides), and do not contain large regions that are not

450 bound by proteins and could form non-specific interactions with nucleolar proteins. It is

451 therefore possible, that in cells lacking TCAB1, TR is strongly retained in the nucleolus

452 while other scaRNAs are less tightly bound, because they lack additional interaction sites

453 with nucleolar proteins. This provides a potential explanation for the phenotypes observed

454 in patients with TCAB1 mutations that suffer from dyskeratosis congenita. The patients

455 have a clear deficiency in telomerase function but no defects in splicing have been

456 reported (Zhong et al., 2011), which would be the consequence of complete loss of 457 scaRNA function and their critical role in spliceosome maturation.

How TCAB1 binding leads to the exclusion of TR and other scaRNA from the

459 nucleolus remains a key unanswered question. TCAB1 interacts with a very short

460 sequence motif in TR, which is far removed from the TR regions that associate with TERT

461 (Fig. S8) (Ghanim et al., 2021). It is therefore unlikely that TCAB1 binding leads to

462 expulsion of TR from the nucleolus by reducing the number of non-specific, multivalent

463 interactions TR can form with nucleolar proteins. As outlined above, we believe that

464 TCAB1 prevents localization of scaRNAs to the nucleolus, rather than extracting

465 scaRNAs that are already localized to the DFC. One potential explanation is that TCAB1

466 counteracts scaRNA recruitment to the nucleolus by inhibiting the nucleolar localization

467 signals within dyskerin (Heiss et al., 1999). Dissecting the molecular mechanism by which

468 TCAB1 leads to exclusion of TR from the nucleolus in future studies will undoubtably shed

469 light on the fundamental principles RNP recruitment to non-membrane bound organelles

470 and its physiological role in cell biology.

471 


\section{Acknowledgements}

473 We would like to thank members of the Schmidt lab and J. Nandakumar for discussions

474 and critical reading of the manuscript and Luke Lavis (HHMI Janelia Research Campus)

475 for providing HaloTag dyes. This work was supported by a grant from the NIH (R00

476 GM120386) to J.C.S. J.C.S. is a Damon Runyon Dale F. Frey Scientist supported (in part)

477 by the Damon Runyon Cancer Research Foundation (DFS-24-17). S.B.C. acknowledges

478 sustained support from the Ernest \& Piroska Major Foundation.

480 Author contributions

481 B.S.A.-M. carried out IF-FISH experiments, telomerase purifications, analyzed 482 telomerase assembly, generated mCherry- and 3xFLAG-HaloTag-dyskerin plasmids, 483 determined their sub-cellular localization, and edited the manuscript. G.I.P. maintained 484 cell lines, established TCAB1 and TR knockout cell lines and carried out IF-FISH 485 experiments. K.A.-B. assisted in establishing the TR knockout cell line and carried out 486 characterization of the TR knockout cells. S.B.C. purified and characterized the anti-TERT 487 sheep antibody. L.H. and K.Y. characterized TCAB1 knock-out clones using Southern 488 blots. J.C.S. carried out all other experiments, designed the research, analyzed data, and 489 wrote the manuscript.

\section{Competing interests}

492 The authors declare no competing interests. 


\section{Materials and Methods}

\section{Cell Lines and Tissue Culture}

495 All cell lines were based on HeLa-EM2-11ht (Weidenfeld et al., 2009) and were cultured 496 in Dulbecco's Modified Eagle Medium including L-glutamine (Gibco) supplemented with

$49710 \%$ fetal bovine serum, $100 \mathrm{units} / \mathrm{ml}$ penicillin and $100 \mu \mathrm{g} / \mathrm{ml}$ streptomycin at $37^{\circ} \mathrm{C}$ with

$4985 \% \mathrm{CO}_{2}$. Live cell imaging was carried out using $\mathrm{CO}_{2}$ independent media supplemented 499 with 2 mM GlutaMAX (Life Technologies), 10\% fetal bovine serum, 100 units/ml penicillin 500 and $100 \mu \mathrm{g} / \mathrm{ml}$ streptomycin at $37^{\circ} \mathrm{C}$ with $5 \% \quad \mathrm{CO}_{2}$. For single-molecule imaging of 501 HaloTag-TERT cell were cultured in homemade imaging dishes made by gluing $22 \times 22$ $502 \mathrm{~mm}$ Nexterion coverslips $(170 \pm 5 \mu \mathrm{m}$, Schott) onto the bottom of plastic $3.5 \times 1.0 \mathrm{~cm}$ cell 503 culture dishes with a hole in the middle using an epoxy adhesive. Prior to chamber

504 assembly the coverslips were washed with $1 \mathrm{M} \mathrm{KOH}$ and $100 \%$ for 30 min each in a 505 sonicating water bath. To enrich for cells in S-phase for live cell imaging experiments, 506 cultures we treated with complete media including $2 \mathrm{mM}$ thymidine for a minimum of 16 507 hours. Cells were released 2 hours prior to imaging by replacing the thymidine containing 508 media with fresh media without thymidine. Puromycin selection was carried out at a 509 concentration of $1 \mu \mathrm{g} / \mathrm{ml}$.

\section{Plasmid Construction and Genome Editing}

512 All plasmids were generated by Gibson assembly (NEB) using standard protocols or by 513 inverse PCR. All plasmids will be made available on Addgene. All Cas9 and sgRNA 514 expression plasmids were based on pX330 (Cong et al., 2013). The homologous 515 recombination donor for the TR knockout was generated by assembling the genomic 
516 sequences immediately upstream and downstream ( 500 bp each) of the TR sequence

517 flanking a puromycin resistance cassette into Hpal linearized pFastBac. The 3xFLAG-

518 HaloTag-NLS plasmids was generated by adding a 3xFLAG-tag to a previously described

519 HaloTag-NLS plasmid (a kind gift from X. Darzacq and A. Hansen) (Hansen et al., 2018).

520 The 3xFLAG-HaloTag-dyskerin plasmid was generated by replacing TERT in our

521 previously described 3xFLAG-HaloTag-TERT expression plasmid with the dyskerin

522 coding sequence (Schmidt et al., 2016). The mCherry-dyskerin plasmid was generated

523 by replacing TERT in our previously described mCherry-TERT expression plasmid with

524 the dyskerin coding sequence (Schmidt et al., 2014). All transfections were carried out

525 using Lipfectamine 2000 (Invitrogen) using the manufacturer's instructions. TCAB1 was

526 knocked-out using a single sgRNA or two separate sgRNA and Cas9 encoding plasmids

527 that were transfected alongside a GFP-expression plasmid. 24 hours after transfection

528 single-cell clones were sorted using the GFP signal. TCAB1 knock-out clones were

529 screened by PCR and confirmed by western blot, Southern Blotting of the TCAB1 locus

530 and immunofluorescence imaging. TR was knocked out by transfecting two sgRNA

531 plasmids and a homologous recombination donor plasmid. 48 hours after transfection

532 puromycin selection was initiated and 1 week after the initiation of selection single-cell

533 clones were generated by dilution into 96-well plates. TR knock-out was confirmed using

534 PCR and Sanger sequencing, fluorescence in situ hybridization, and RT-qPCR.

\section{Immunofluorescence and Fluorescence In Situ Hybridization Imaging}

537 Fixed cell immunofluorescence imaging and fluorescence in situ hybridization was carried

538 out as previously described (Schmidt et al., 2014). Briefly, cells grown on coverslips were 
539 fixed in PBS supplemented with 4\% formaldehyde. When using the HaloTag for

540 fluorescence detection cells were incubated with 100 nM of JF646 HaloTag-ligand for 30

541 min prior to fixation. Unincorporated ligand was removed by 3 washes with complete

542 media followed by placing the cells back in the incubator for $10 \mathrm{~min}$ to let additional dye

543 leak out of the cells. mEOS3.2-TRF2 was detected using the intrinsic fluorescence of

544 green form of mEOS3.2. After removing the fixation solution using 2 PBS washes,

545 coverslips were transferred into aluminum foil covered humidity chambers with a parafilm

546 layer and rinsed with $1 \mathrm{ml}$ of PBS with $0.2 \%$ Triton $\mathrm{X}-100$. Cells were than incubated in

547 blocking buffer (PBS, 0.2\% Triton X-100, 3\% BSA) for 30 minutes, followed by incubation

548 with primary antibodies diluted in blocking buffer for 1 hour. All primary antibodies (see

549 key resource table) were used at a concentration of $1 \mu \mathrm{g} / \mathrm{ml}$. After three washes with PBS

$550+0.2 \%$ Triton X-100, coverslips were incubated with secondary antibodies diluted in PBS

$551+0.2 \%$ Triton $\mathrm{X}-100$ for 1 hour. All secondary antibodies (see key resource table) were

552 used at a concentration of $4 \mu \mathrm{g} / \mathrm{ml}$. Cells were washed three times PBS $+0.2 \%$ Triton X-

553100 prior to a second fixation with PBS $+4 \%$ formaldehyde. In cases where nuclear

554 staining was used the first of the three washing steps also included $0.1 \mu \mathrm{g} / \mathrm{ml} \mathrm{HOECHST}$.

555 After the second fixation steps coverslips were dehydrates in three steps with ethanol

$556(70 \%, 95 \%, 100 \%)$, re-hydrated in 2xSSC $+50 \%$ formamide, blocked for 1 hour in

557 hybridization buffer $(100 \mathrm{mg} / \mathrm{ml}$ dextran sulfate, $0.125 \mathrm{mg} / \mathrm{ml}$ E. coli tRNA, $1 \mathrm{mg} / \mathrm{ml}$

558 nuclease free BSA, $0.5 \mathrm{mg} / \mathrm{ml}$ salmon sperm DNA, $1 \mathrm{mM}$ vanadyl ribonucleoside 559 complexes, $50 \%$ formamide, $2 \times S S C$ ) at $37^{\circ} \mathrm{C}$, before incubating the coverslips in

560 hybridization buffer supplemented with three TR probes (30 ng per coverslip) over night

561 at $37^{\circ} \mathrm{C}$. Probe sequences were previously described (Tomlinson et al., 2006). After 
562 hybridization coverslips were washes twice for 30 minutes in $2 x S S C+50 \%$ formamide

563 and then mounted on slides using ProLong Antifade Diamond mounting media (Life

564 Technologies). Microscopy was carried out using a DeltaVision Elite microscope using a

565 60x PlanApo objective (1.42 NA) and a pco.edge sCMOS camera. We acquired 20 Z-

566 sections spaced by $0.2 \mu \mathrm{m}$, followed by image deconvolution and maximum intensity

567 projection of the sections using the DeltaVision Softworx software.

569 Single-Molecule Live Cell Imaging

570 Live cell single-molecule imaging was carried out on a Olympus IX83 inverted microscope

571 equipped with a 4-line cellTIRF illuminator (405 nm, $488 \mathrm{~nm}, 561 \mathrm{~nm}, 640 \mathrm{~nm}$ lasers), a

572 Olympus UAPO 100x TIRF objective (1.49 NA), a CAIRN TwinCam beamsplitter, 2 Andor

573 iXon 897 Ultra EMCCD cameras, and a blacked-out environmental control enclosure. The

574 microscope was operated using the Olympus cellSense software. 3xFLAG-HaloTag-

575 TERT was labeled for $2 \mathrm{~min}$ in complete media supplemented with $100 \mathrm{nM}$ JF646-

576 HaloTag ligand (Grimm et al., 2015). After removing the HaloTag-ligand with three

577 washes in complete media, cells were placed back in the incubator for 10 min to allow

578 unincorporated dye to leak out of the cells. Cells were then transferred into $\mathrm{CO}_{2}$

579 independent media and put on the microscope which was heated to $37^{\circ} \mathrm{C}$. Single-

580 molecule imaging was carried out at 50 or 100 frames per second using highly inclined

581 laminated optical sheet illumination (Tokunaga et al., 2008). Movies were typically 20

582 seconds in length (2000 frames) and were followed by a transmitted light acquisition to

583 visualize overall cell morphology. 


\section{RT-qPCR}

586 RNA samples for RT-qPCR analysis were generated by using RNA mini prep kits

587 (Zymogen) using 2 million cells as starting material. Reverse transcription was carried 588 out using random hexamer primers and SuperScript III reverse transcriptase (Invitrogen)

589 according to the manufacturer's instructions. qPCR was carried out using the Maxima 590 SYBR Green qPCR master mix (Thermo Scientific) using primers for GAPDH and TR 591 according to the manufacturer's instructions. All qPCR reactions were carried out in 592 triplicates and three independent biological replicates were analyzed.

\section{Southern Blotting}

595 Southern blotting was carried out using standard protocols (Southern, 2006). Briefly, 596 genomic DNA generated by phenol-chloroform extraction after cell lysis using TE 597 supplemented with $0.5 \%$ SDS and $0.1 \mathrm{mg} / \mathrm{ml}$ Proteinase $\mathrm{K}$, was digested with BamHI 598 (generating a 1394 bp fragment spanning exons 1-3 of the TCAB1 locus) and separated 599 on a $0.8 \%$ agarose gel. The DNA was then transferred on a Hybond-N+ nylon membrane 600 using capillary transfer. The TCAB1 locus was detected using radioactive probes (alpha-

$\left.601 \quad{ }^{32} \mathrm{P}-\mathrm{dCTP}\right)$ generated by randomly primed DNA synthesis using an 800 bp PCR product 602 overlapping with the $1394 \mathrm{bp}$ restriction fragment as a template and Klenow polymerase 603 (NEB).

604

605 Western Blotting

606 Mini-PROTEAN TGX stain-free gels (Bio-Rad) were used for SDS-PAGE. Total protein 607 was detected using a ChemiDoc MP (Bio-Rad) after a 45 second UV activation. Western 
608 transfer was carried out using the Trans-Blot Turbo transfer system (Bio-Rad) according

609 to the manufacturer's instructions using the mixed molecular weight transfer setting.

610 Immuno-blotting was carried out using standard protocols. The TCAB1 antibody

611 (Proteintech) was used at a 1:2000 dilution, the TERT antibody (Abcam) was used at a

612 1:4000 dilution, and the dyskerin antibody (Santa Cruz Biotech) was used at a 1:200

613 dilution. Secondary antibodies were used at a 1:10000 dilution.

615 Northen Blotting

616 RNA was extracted from cell lysates and purified telomerase samples supplemented with

$61710 \mathrm{ng}$ of a loading and recovery control (in vitro transcribed TR 34-328) using the RNeasy

618 Mini kit (Qiagen) and eluted in 30 ul of RNase free water. 15 ul of eluted RNA was mixed

619 with 15 ul of $2 x$ formamide loading buffer (0.1XTBE, 25 mM EDTA, 0.1\% bromophenol

620 blue, $0.1 \%$ xylene cyanol, $93 \%$ formamide) and heated to $60{ }^{\circ} \mathrm{C}$ for $5 \mathrm{~min}$. Samples were

621 separated on a 6\% TBE, 7M Urea, polyacrylamide gel (Life Technologies), and

622 transferred to a Hybond $\mathrm{N}+$ membrane (Cytiva) using a wet-blotting apparatus in 1x TBE

623 for 2 hours at $0.5 \mathrm{~A}$ of constant current in the cold room. After transfer, membranes were

624 UV-crosslinked, and pre-hybridized in Church buffer for 2 hours at $50{ }^{\circ} \mathrm{C}$. Three DNA

625 oligos complementary to TR were radioactively labeled using T4 PNK (NEB) and $\sim 10 \times 10^{6}$

$626 \mathrm{cpm}$ of each probe were added to the membrane. Hybridization was carried out at $50{ }^{\circ} \mathrm{C}$

627 overnight. Membranes were washed three times with 2xSSC, $0.1 \%$ SDS prior to exposure

628 to a storage phosphorescence screen (Cytiva) which was then imaged on an Amersham

629 Typhoon IP phosphoimager (Cytiva). 


\section{Telomerase Expression and Purification}

632 Cell lines were transfected in $15 \mathrm{~cm}$ tissue culture plates at $\sim 90 \%$ confluency $\left(\sim 25-30 \times 10^{6}\right.$

633 cells) using $7.5 \mu \mathrm{g}$ of TERT plasmid, $30 \mu \mathrm{g}$ of TR plasmid and $75 \mu \mathrm{l}$ of Lipofectamine 2000

634 in 1875 ul of Opti-MEM (Cristofari and Lingner, 2006). Transfected cells were split to three

$63515 \mathrm{~cm}$ dishes 24 hours after transfection. 48 hours after transfection cells were counted,

636 harvested, and snap frozen in liquid nitrogen. Cells were lysed in $1 \mathrm{ml}$ of CHAPS lysis

637 buffer supplemented with $5 \mu$ of RiboLock RNase inhibitor (10 mM TRIS pH 7.5, $1 \mathrm{mM}$

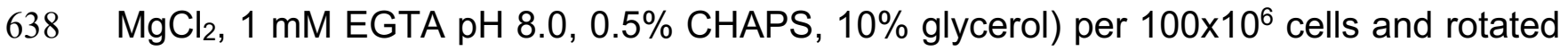

639 at $4{ }^{\circ} \mathrm{C}$ for $30 \mathrm{~min}$. Lysates were cleared in a table-top centrifuge at 21,000xg for $15 \mathrm{~min}$

640 at $4{ }^{\circ} \mathrm{C}$. Identical cell equivalents were used for all samples. $45 \mu \mathrm{g}$ of anti-TERT antibody

641 was added per $\mathrm{ml}$ of cleared lysate and samples were rotated for 1 hour at $4{ }^{\circ} \mathrm{C}$. Lysates

642 were then added to $100 \mu \mathrm{l}$ of protein $\mathrm{G}$ agarose and rotated for 1 hour at $4^{\circ} \mathrm{C}$. The resin 643 was spun down at 1000xg and washed four times with $1 \mathrm{ml}$ of Buffer W (20 mM HEPES $644 \mathrm{pH}$ 7.9, $300 \mathrm{mM} \mathrm{KCl}, 2$ mM MgCl 2,1 mM EDTA, 1 mM DTT, 1 mM PMSF, 0.1\% Triton X$645100,10 \%$ glycerol). TERT was eluted in $100 \mu$ of Buffer W supplemented with $5 \mu$ of 1 mM TERT peptide by rotating for $30 \mathrm{~min}$ at room temperature.

648 Telomerase Activity Assays

649 Telomerase assays were carried out in $20 \mu \mathrm{l}$ of reaction buffer (50 mM TRIS pH 8.0, 150 $650 \mathrm{mM} \mathrm{KCl}, 1 \mathrm{mM} \mathrm{MgCl}$, 2 mM DTT, 100 nM TTAGGGTTAGGGTTAGG oligo, $10 \mu \mathrm{M}$ dATP, $65110 \mu \mathrm{M}$ dGTP, $10 \mu \mathrm{M}$ dTTP, $0.165 \mu \mathrm{M}$ dGTP [ $\alpha-32 \mathrm{P}] 3000 \mathrm{Ci} / \mathrm{mmol})$ including $2 \mu \mathrm{l}$ of 652 purified telomerase for 1 hour at $30{ }^{\circ} \mathrm{C}$. Telomerase was incubated with the substrate 653 oligo for $15 \mathrm{~min}$ at room temperature, prior to initiating the reaction by addition of dNTPs. 
654 Reactions were stopped by adding $100 \mu \mathrm{l}$ of $3.6 \mathrm{M}$ of ammonium acetate supplemented

655 with $20 \mathrm{\mu g}$ of glycogen and 32P 5'-end labeled loading control oligos

656 (TTAGGGTTAGGGTTAGGG, TTAGGGTTAGGGTTAG). Reaction products were

657 precipitated using $500 \mu \mathrm{l}$ of ice-cold ethanol and stored at $-20{ }^{\circ} \mathrm{C}$ over-night. Reaction

658 products were spun down in a table-top centrifuge at max speed for 30 min at $4{ }^{\circ} \mathrm{C}$,

659 washed with $500 \mu \mathrm{l}$ of $70 \%$ ethanol, and spun down again speed for 30 min at $4{ }^{\circ} \mathrm{C}$. The

$66070 \%$ ethanol was decanted, and the reaction products were dried in an Eppendorf

661 vacuum concentrator at $45^{\circ} \mathrm{C}$. Reaction products were resuspended in $20 \mu$ lof loading

662 buffer (0.05XTBE, 25 mM EDTA, 0.05\% bromophenol blue, 0.05\% xylene cyanol, 46.5\%

663 formamide) and incubated at $95^{\circ} \mathrm{C}$ for $5 \mathrm{~min} .10 \mu \mathrm{l}$ of each sample was separated on a

$66412 \%$ polyacrylamide, $7 \mathrm{M}$ urea sequencing gel pre-run for $45 \mathrm{~min}$ at $90 \mathrm{~W}$. Gels were dried

665 and exposed to a storage phosphorescence screen (Cytiva) and imaged on an

666 Amersham Typhoon IP phosphoimager (Cytiva).

668 Single-Particle Tracking

669 Single-particle tracking was carried out in MATLAB 2019a using a batch parallel670 processing version of SLIMfast modified to allow the input of TIFF files (kindly provided 671 by Xavier Darzacq and Anders Hansen) (Hansen et al., 2018), an implementation of the 672 Multiple-Target-Tracing algorithm (Sergé et al., 2008), with the following settings: 673 Exposure Time $=10 \mathrm{~ms}, \mathrm{NA}=1.49$, Pixel Size $=0.16 \mu \mathrm{m}$, Emission Wavelength $=664$

$674 \mathrm{~nm}, \mathrm{D}_{\max }=5 \mu \mathrm{m}^{2} / \mathrm{s}$, Number of gaps allowed $=2$, Localization Error $=10^{-5}$, Deflation

675 Loops $=0$. Diffusion coefficients and the fraction of molecules in each distinct particle 676 population were determined using the MATLAB version of the Spot-On tool (kindly 
677 provided by Xavier Darzacq and Anders Hansen) (Hansen et al., 2018) with the following

678 settings: TimeGap $=10 \mathrm{~ms}$ or $20 \mathrm{~ms}, \mathrm{dZ}=0.700 \mu \mathrm{m}$, GapsAllowed $=2$, TimePoints $=8$,

679 JumpsToConsider $=4$, BinWidth $=0.01 \mu \mathrm{m}$, PDF-fitting, D_Free1_3State $=\left[\begin{array}{ll}1 & 25\end{array}\right]$,

680

D_Free2_3State $=[0.11]$, D_Bound_3State $=[0.00010 .1]$. For all experiments we carried

681 out 3 independent biological replicates with at least 15 cells for each cell line. The

682 complete data set can be found in supplemental figure S6D. The statistical significance

683 of differences in particle fractions and diffusion coefficients were assessed using a two-

684 tailed T-Test.

685

\section{Quantification of Fixed Cell Imaging}

687 For the quantification of cellular TR distribution in control and TCAB1 knockout cells we

688 assigned cells into one of three categories: Cells with TR only at telomeres, Cells with TR

689 only in nucleoli, Cells with TR at telomeres and in nucleoli. We carried out 3 independent

690 biological replicates and counted a minimum of 100 cells for control and TCAB1 knockout

691 cells.

692

693 Quantification of RT-qPCR data

694 RT-qPCR experiments were carried out in triplicate and the TR Ct value was normalized

695 to the GAPDG $\mathrm{Ct}$ value. The mean $\triangle \mathrm{Ct}$ (Ct of TR - Ct of GAPDH) value from three

696 independent experiments and the corresponding standard deviation is shown in figure

697 S3B.

698

699

Quantification of Western Blots, Northern Blots, and Telomerase Activity Assays 
700 Gel images from Western Blots, Northern Blots, and Telomerase Activity Assays were

701 analyzed using ImageQuant TL 8.2. To quantify TR levels in Northern blots the TR band

702 intensity was normalized to the loading and recovery control signal added to the RNA

703 sample prior to RNA purification. To quantify telomerase activity the whole lane intensity

704 starting at repeat 1 was determined and divided by the sum of the loading control signals.

705 Telomerase processivity was calculated by dividing product intensity $>7$ repeats by the

706 total signal in the respective lane. The statistical significance of the observed differences

707 was calculated using a two-tailed T-test using a minimum of three biological replicates.

708 Each biological replicate (independent telomerase expression and purification) was

709 analyzed in technical triplicate.

710

711 


\section{References}

713

714

715

716

\section{7}

718

719

720

721

722

723

724

725

726

727

728

729

730

731

732

733

734

735

736

737

738

739

740

741

742

743

744
Angrisani A, Vicidomini R, Turano M, Furia M. 2014. Human dyskerin: beyond telomeres. Biol Chem 395:593-610. doi:10.1515/hsz-2013-0287

Armanios M, Blackburn EH. 2012. The telomere syndromes. Nat Rev Genet 13:693 704. doi:10.1038/nrg3246

Chen L, Roake CM, Freund A, Batista PJ, Tian S, Yin YA, Gajera CR, Lin S, Lee B, Pech MF, Venteicher AS, Das R, Chang HY, Artandi SE. 2018. An Activity Switch in Human Telomerase Based on RNA Conformation and Shaped by TCAB1. Cell 174. doi:10.1016/j.cell.2018.04.039

Cohen SB, Graham ME, Lovrecz GO, Bache N, Robinson PJ, Reddel RR. 2007. Protein Composition of Catalytically Active Human Telomerase from Immortal Cells. Science 315:1850-1853. doi:10.1126/science.1138596

Cong L, Ran FA, Cox D, Lin S, Barretto R, Habib N, Hsu PD, Wu X, Jiang W, Marraffini LA, Zhang F. 2013. Multiplex Genome Engineering Using CRISPR/Cas Systems. Science 339:819-823. doi:10.1126/science.1231143

Cristofari G, Lingner J. 2006. Telomere length homeostasis requires that telomerase levels are limiting. Embo J 25:565 574. doi:10.1038/sj.emboj.7600952

Darzacq X, Kittur N, Roy S, Shav-Tal Y, Singer RH, Meier UT. 2006. Stepwise RNP assembly at the site of H/ACA RNA transcription in human cells. J Cell Biology 173:207-218. doi:10.1083/jcb.200601105

Freund A, Zhong FL, Venteicher AS, Meng Z, Veenstra TD, Frydman J, Artandi SE. 2014. Proteostatic Control of Telomerase Function through TRiC-Mediated Folding of TCAB1. Cell 159:1389 1403. doi:10.1016/j.cell.2014.10.059

Gallardo F, Olivier C, Dandjinou AT, Wellinger RJ, Chartrand P. 2008. TLC1 RNA nucleo-cytoplasmic trafficking links telomerase biogenesis to its recruitment to telomeres. Embo J 27:748 757. doi:10.1038/emboj.2008.21

Ghanim GE, Fountain AJ, Roon A-MM van, Rangan R, Das R, Collins K, Nguyen THD. 2021. Structure of human telomerase holoenzyme with bound telomeric DNA. Nature 1-5. doi:10.1038/s41586-021-03415-4

Grimm JB, English BP, Chen J, Slaughter JP, Zhang Z, Revyakin A, Patel R, Macklin JJ, Normanno D, Singer RH, Lionnet T, Lavis LD. 2015. A general method to improve fluorophores for live-cell and single-molecule microscopy. Nat Methods 12:244 50-3 p following 250. doi:10.1038/nmeth.3256 
Hansen AS, Woringer M, Grimm JB, Lavis LD, Tjian R, Darzacq X. 2018. Robust model-based analysis of single-particle tracking experiments with Spot-On. Elife 7. doi:10.7554/elife.33125

Heiss NS, Girod A, Salowsky R, Wiemann S, Pepperkok R, Poustka A. 1999. Dyskerin Localizes to the Nucleolus and Its Mislocalization Is Unlikely to Play a Role in the Pathogenesis of Dyskeratosis Congenita. Hum Mol Genet 8:2515-2524. doi:10.1093/hmg/8.13.2515

Hernandez-Verdun D. 2014. Assembly and disassembly of the nucleolus during the cell cycle. Nucleus 2:189-194. doi:10.4161/nucl.2.3.16246

Hyman AA, Weber CA, Jülicher F. 2014. Liquid-Liquid Phase Separation in Biology. Annu Rev Cell Dev Bi 30:39-58. doi:10.1146/annurev-cellbio-100913-013325

Jády BE, Bertrand E, Kiss T. 2004. Human telomerase RNA and box H/ACA scaRNAs share a common Cajal body-specific localization signal. J Cell Biology 164:647-652. doi:10.1083/jcb.200310138

Lafontaine DLJ, Riback JA, Bascetin R, Brangwynne CP. 2021. The nucleolus as a multiphase liquid condensate. Nat Rev Mol Cell Bio 22:165-182. doi:10.1038/s41580-020-0272-6

Mitrea DM, Kriwacki RW. 2016. Phase separation in biology; functional organization of a higher order. Cell Commun Signal Ccs 14:1. doi:10.1186/s12964-015-0125-7

Nagpal N, Wang J, Zeng J, Lo E, Moon DH, Luk K, Braun RO, Burroughs LM, Keel SB, Reilly C, Lindsley RC, Wolfe SA, Tai AK, Cahan P, Bauer DE, Fong YW, Agarwal S. 2020. Small-Molecule PAPD5 Inhibitors Restore Telomerase Activity in Patient Stem Cells. Cell Stem Cell 26:896-909.e8. doi:10.1016/j.stem.2020.03.016

Nandakumar J, Bell CF, Weidenfeld I, Zaug AJ, Leinwand LA, Cech TR. 2012. The TEL patch of telomere protein TPP1 mediates telomerase recruitment and processivity. Nature 492:285 289. doi:10.1038/nature11648

Nandakumar J, Cech TR. 2013. Finding the end: recruitment of telomerase to telomeres. Nat Rev Mol Cell Bio 14. doi:10.1038/nrm3505

Riback JA, Zhu L, Ferrolino MC, Tolbert M, Mitrea DM, Sanders DW, Wei M-T, Kriwacki RW, Brangwynne CP. 2020. Composition-dependent thermodynamics of intracellular phase separation. Nature 581:209-214. doi:10.1038/s41586-020-2256-2

Schmidt JC, Cech TR. 2015. Human telomerase: biogenesis, trafficking, recruitment, and activation. Gene Dev 29:1095 1105. doi:10.1101/gad.263863.115 
Schmidt JC, Dalby AB, Cech TR. 2014. Identification of human TERT elements necessary for telomerase recruitment to telomeres. Elife 3:e03563. doi:10.7554/elife.03563

Schmidt JC, Zaug AJ, Cech TR. 2016. Live Cell Imaging Reveals the Dynamics of Telomerase Recruitment to Telomeres. Cell 166:1188 1197.e9. doi:10.1016/j.cell.2016.07.033

Schmidt JC, Zaug AJ, Kufer R, Cech TR. 2018. Dynamics of human telomerase recruitment depend on template-telomere base pairing. Mol Biol Cell 29:869 880 . doi:10.1091/mbc.e17-11-0637

Sergé A, Bertaux N, Rigneault H, Marguet D. 2008. Dynamic multiple-target tracing to probe spatiotemporal cartography of cell membranes. Nat Methods 5:687 694. doi:10.1038/nmeth.1233

Shukla S, Jeong H-C, Sturgeon CM, Parker R, Batista LFZ. 2020. Chemical inhibition of PAPD5/7 rescues telomerase function and hematopoiesis in dyskeratosis congenita. Blood Adv 4:2717-2722. doi:10.1182/bloodadvances.2020001848

Shukla S, Schmidt JC, Goldfarb KC, Cech TR, Parker R. 2016. Inhibition of telomerase RNA decay rescues telomerase deficiency caused by dyskerin or PARN defects. Nat Struct Mol Biol 23:286 292. doi:10.1038/nsmb.3184

Southern E. 2006. Southern blotting. Nat Protoc 1:518-525. doi:10.1038/nprot.2006.73

Stern JL, Zyner KG, Pickett HA, Cohen SB, Bryan TM. 2012. Telomerase Recruitment Requires both TCAB1 and Cajal Bodies Independently. Mol Cell Biol 32:2384-2395. doi:10.1128/mcb.00379-12

Stewart SA, Weinberg RA. 2006. Telomeres: Cancer to Human Aging. Annu Rev Cell Dev Bi 22:531-557. doi:10.1146/annurev.cellbio.22.010305.104518

Stuart BD, Choi J, Zaidi S, Xing C, Holohan B, Chen R, Choi M, Dharwadkar P, Torres F, Girod CE, Weissler J, Fitzgerald J, Kershaw C, Klesney-Tait J, Mageto Y, Shay JW, Ji W, Bilguvar K, Mane S, Lifton RP, Garcia CK. 2015. Exome sequencing links mutations in PARN and RTEL1 with familial pulmonary fibrosis and telomere shortening. Nat Genet 47:512 517. doi:10.1038/ng.3278

Tokunaga M, Imamoto N, Sakata-Sogawa K. 2008. Highly inclined thin illumination enables clear single-molecule imaging in cells. Nat Methods 5:159 161. doi:10.1038/nmeth1171

Tomlinson RL, Ziegler TD, Supakorndej T, Terns RM, Terns MP. 2006. Cell cycleregulated trafficking of human telomerase to telomeres. Mol Biol Cell 17:955 965. doi:10.1091/mbc.e05-09-0903 
813 Tseng C-K, Wang H-F, Burns AM, Schroeder MR, Gaspari M, Baumann P. 2015. Human Telomerase RNA Processing and Quality Control. Cell Reports 13:22322243. doi:10.1016/j.celrep.2015.10.075

Tummala H, Walne A, Collopy L, Cardoso S, Fuente J de la, Lawson S, Powell J, Cooper N, Foster A, Mohammed S, Plagnol V, Vulliamy T, Dokal I. 2015. Poly(A)specific ribonuclease deficiency impacts telomere biology and causes dyskeratosis congenita. J Clin Invest 125:2151-2160. doi:10.1172/jci78963

Venteicher AS, Abreu EB, Meng Z, McCann KE, Terns RM, Veenstra TD, Terns MP, Artandi SE. 2009. A human telomerase holoenzyme protein required for Cajal body localization and telomere synthesis. Science 323:644 648. doi:10.1126/science.1165357

Vogan JM, Zhang X, Youmans DT, Regalado SG, Johnson JZ, Hockemeyer D, Collins K. 2016. Minimized human telomerase maintains telomeres and resolves endogenous roles of H/ACA proteins, TCAB1, and Cajal bodies. Elife 5:693. doi:10.7554/elife.18221

Weidenfeld I, Gossen M, Löw R, Kentner D, Berger S, Görlich D, Bartsch D, Bujard H, Schönig K. 2009. Inducible expression of coding and inhibitory RNAs from retargetable genomic loci. Nucleic Acids Res 37:e50. doi:10.1093/nar/gkp108

Wu RA, Upton HE, Vogan JM, Collins K. 2017. Telomerase Mechanism of Telomere Synthesis. Annu Rev Biochem 86:439 460. doi:10.1146/annurev-biochem-061516045019

Xi L, Cech TR. 2014. Inventory of telomerase components in human cells reveals multiple subpopulations of hTR and hTERT. Nucleic Acids Res 42:8565-8577. doi:10.1093/nar/gku560

Yao R-W, Xu G, Wang Ying, Shan L, Luan P-F, Wang Yang, Wu M, Yang L-Z, Xing YH, Yang L, Chen L-L. 2019. Nascent Pre-rRNA Sorting via Phase Separation Drives the Assembly of Dense Fibrillar Components in the Human Nucleolus. Mol Cell 76:767-783.e11. doi:10.1016/j.molcel.2019.08.014

Zhong F, Savage SA, Shkreli M, Giri N, Jessop L, Myers T, Chen R, Alter BP, Artandi SE. 2011. Disruption of telomerase trafficking by TCAB1 mutation causes dyskeratosis congenita. Gene Dev 25:11-16. doi:10.1101/gad.2006411 OB-Fold Domain Controls Telomere Maintenance by Recruiting Telomerase to Chromosome Ends. Cell 150:481 494. doi:10.1016/j.cell.2012.07.012 


\section{Supplementary Information}

A

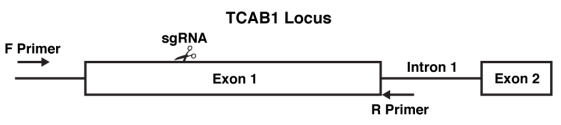

B

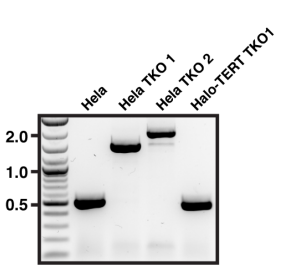

C Halo-TERT TKO1 (25 bp deletion)
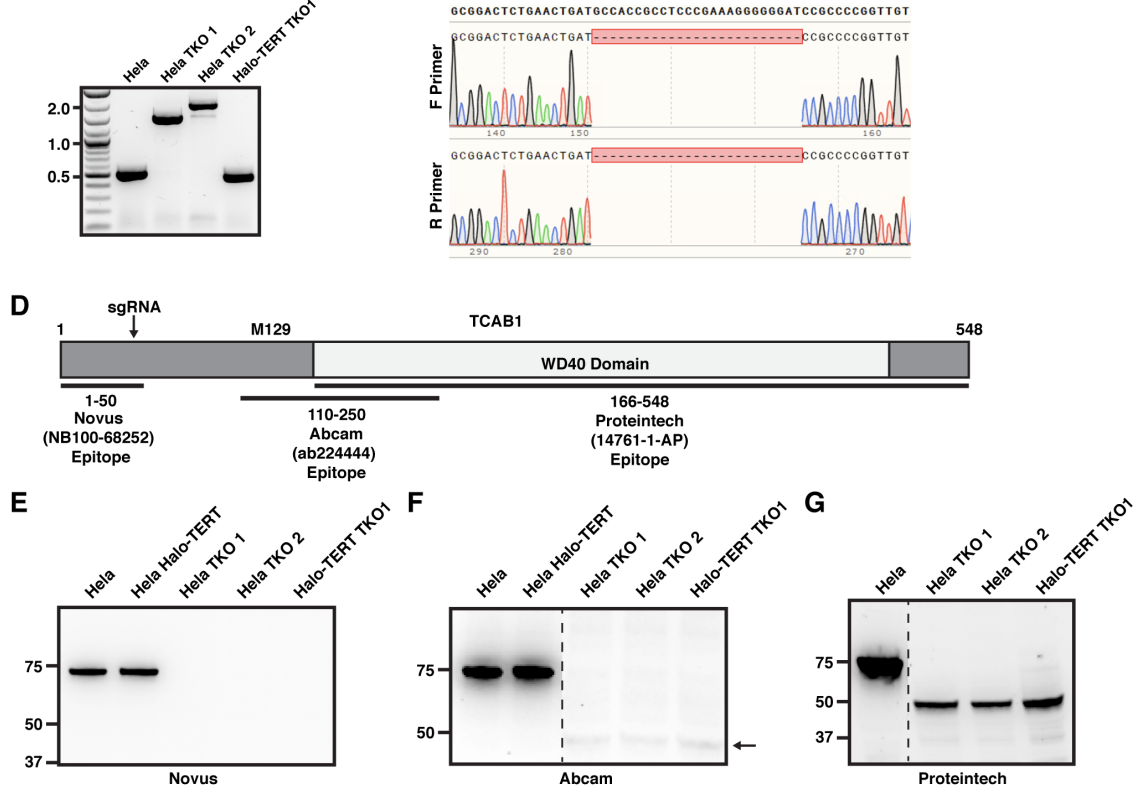

H

I
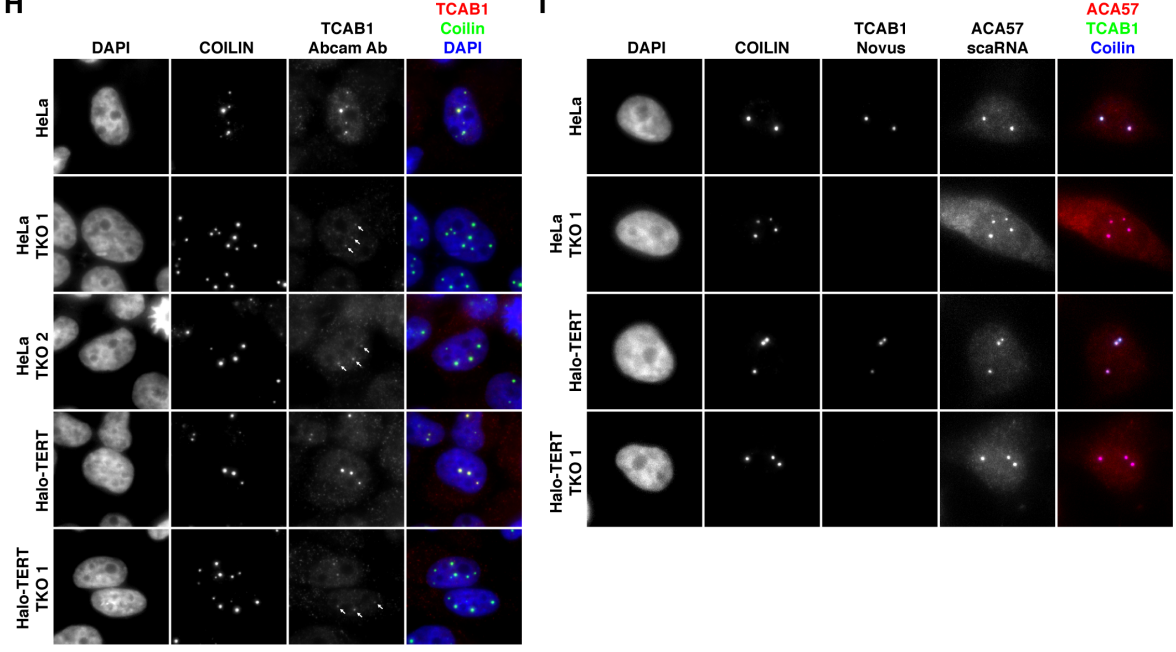

Figure 1 - Figure Supplement 1. (A) Strategy to knock-out TCAB1 with a single Cas 9 and a single sgRNA targeting exon 1 of the TCAB1 gene. (B) PCR of TCAB1 knock-out clones using the primers indicated in (A) demonstrating the absence of a WT TCAB1 allele. (C) Sanger sequencing of the PCR product derived from Halo-TERT TKO1 (B) demonstrating deletion causing a frame shift. (D) Model of TCAB1 protein indicating the antigens used to raise the indicated TCAB1 antibodies. (E-G) Western blots of parental cells and TCAB1 knock-out clones using (E) the Novus Biological, (F) Abcam, and (G) Proteintech TCAB1 antibodies, demonstrating the presence of a truncated TCAB1 protein in the putative TCAB1 knock-out cells. (H) IF using antibodies against coilin and TCAB1 (Abcam) demonstrating that truncated TCAB1 can be detected in Cajal bodies (white arrows) in putative TCAB1 knock-out clones. (I) IF using coilin and TCAB1 (Novus Biologicals) antibodies coupled to FISH with probes against the scaRNA ACA57, demonstrating that the Novus Biologicals antibody fails to detect the truncated TCAB1 and scaRNA localization is unchanged in putative TCAB1 knock-out cells. 
A

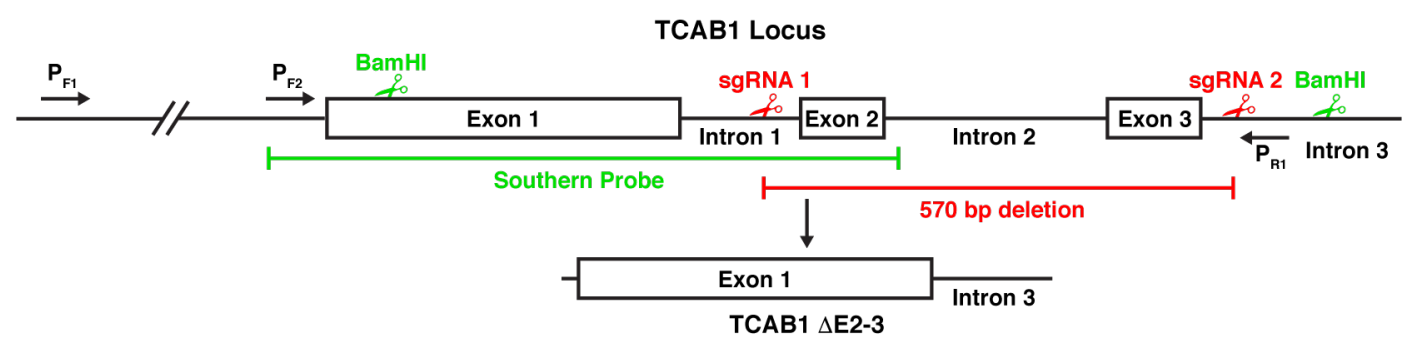

B
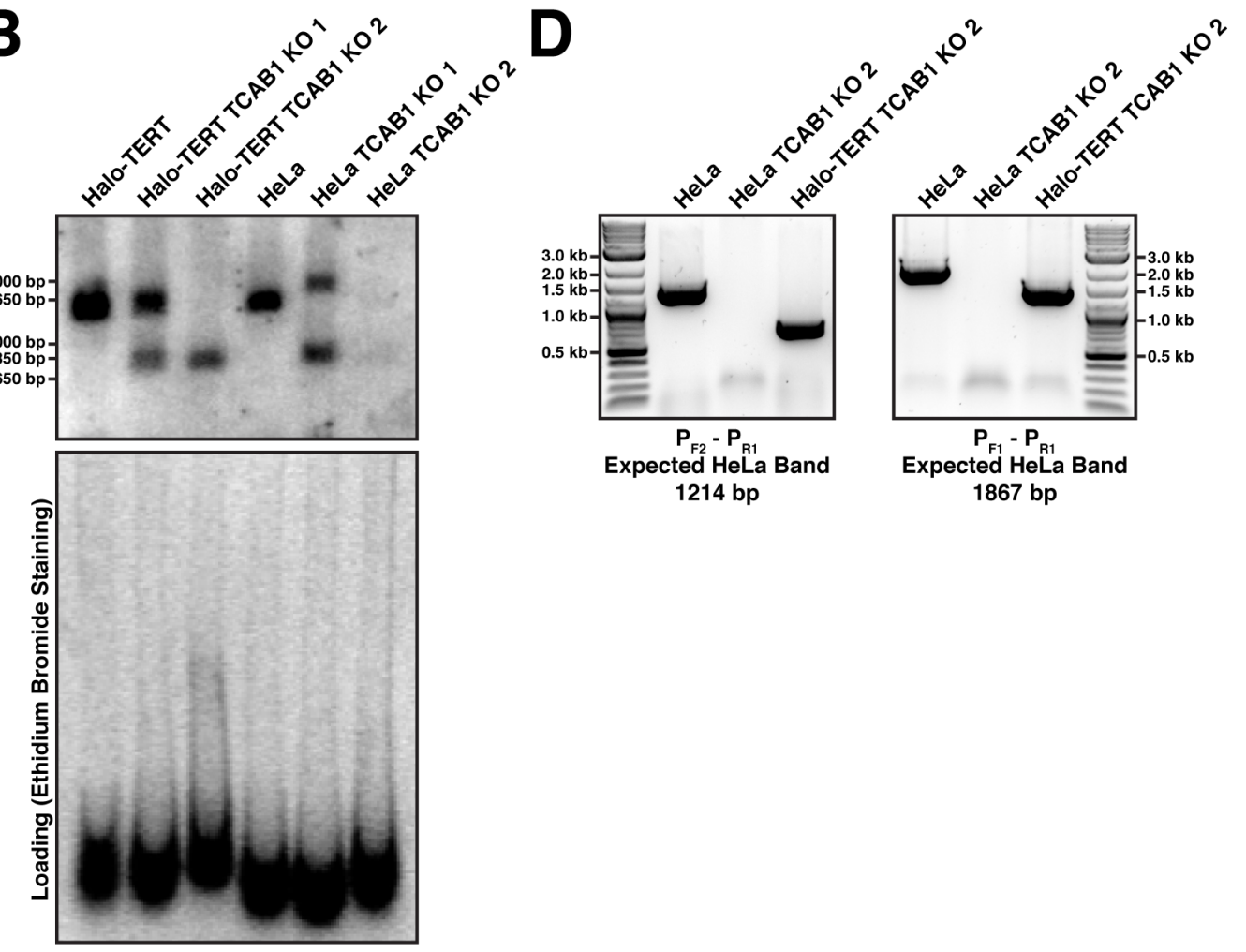

1214 bp

Expected HeLa Band

D
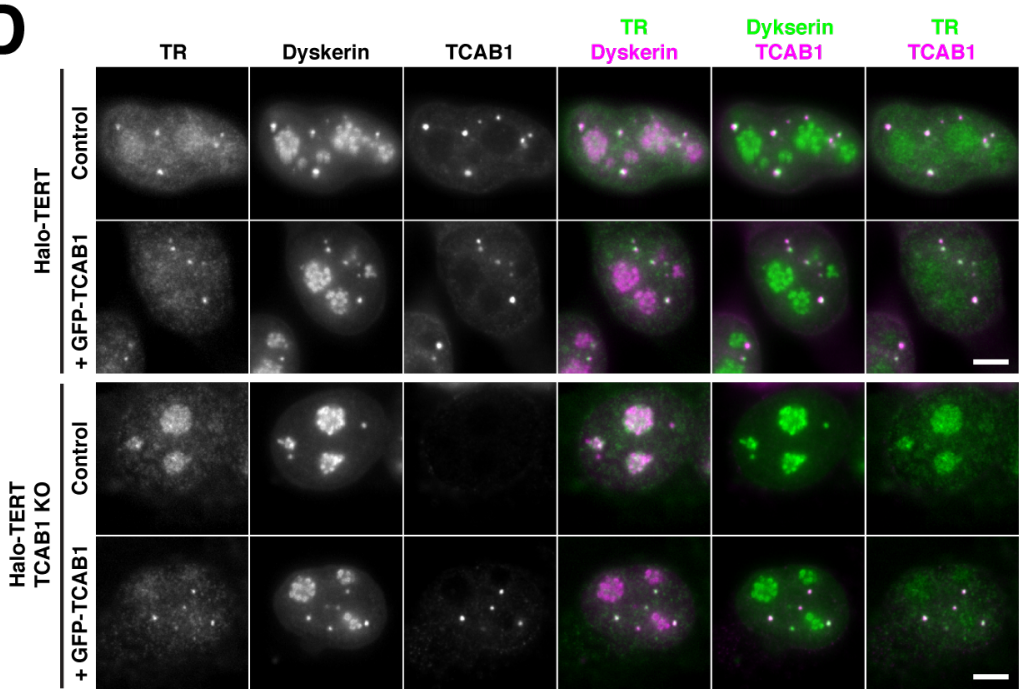

Figure 1 - Figure Supplement 2. (A) Strategy to knock-out TCAB1 using Cas9 and two sgRNAs targeting introns 1 and 3. (B) Southern blot of genomic DNA digested with BamHI from parental cells and TCAB1 knock-out clones using a probes generated from a PCR product of the TCAB1 gene indicated in (A) demonstrating the expected truncation of the TCAB1 gene in Halo-TERT TCAB1 KO 2. HeLa TCAB1 

available under aCC-BY-NC-ND 4.0 International license.

KO 2 carries larger deletions completely removing exons 1 and 2 from the TCAB1 gene. (C) PCR using primers indicated in (A) of genomic DNA from parental cells and TCAB1 knock-out clones confirming the deletion of critical regions of the TCAB1 gene show in (B). (D) Immuno-fluorescence with anti-dyskerin and anti-TCAB1 antibodies coupled to fluorescence in-situ hybridization with probes against TR, demonstrating that expression of GFP-TCAB1 in TCAB1 knockout cells rescues TR localization to Cajal bodies (scale bar $=5 \mu \mathrm{m}$ ).

A

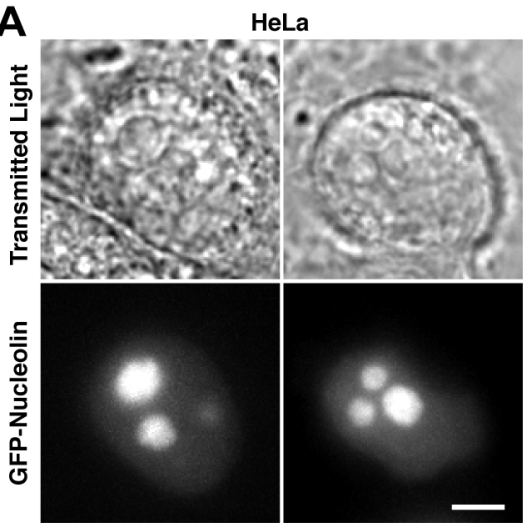

B HeLa

C

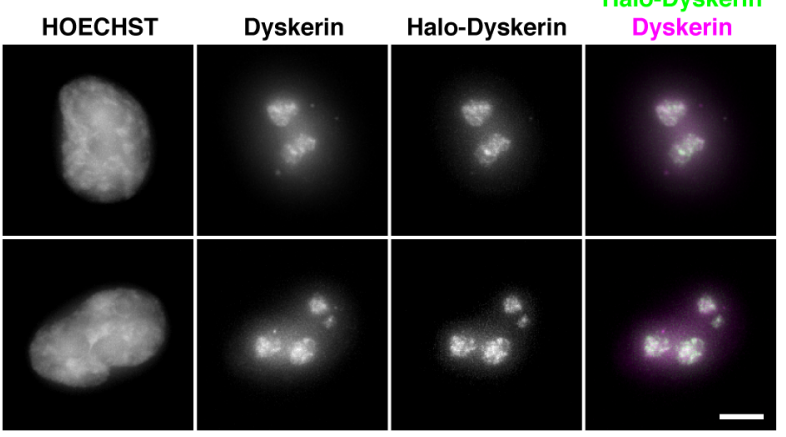

Figure 2 - Figure Supplement 1. (A) Images of HeLa cells transiently expressing GFP-nucleolin to mark nucleoli. The GFP-nucleolin signal overlaps with circular shapes visible under transmitted light illumination (scale bar $=2 \mu \mathrm{m}$ ). (B) Images of HeLa cells transiently expressing GFP-nucleolin and 3xFLAG-HaloTagNLS labeled with JF646. The 3xFLAG-HaloTag-NLS signal (maximum intensity projection of 1000 frames of a single-molecule imaging movie) clearly overlaps with the GFP-nucleolin signal (red dashed outline), demonstrating that 3xFLAG-HaloTag-NLS can enter the nucleolus (scale bar $=2 \mu \mathrm{m}$ ). (C) Images of HeLa cells transiently expressing 3xFLAG-HaloTag-dyskerin labeled with JF646 and probed with an antibody against dyskerin, demonstrating that 3xFLAG-HaloTag-dyskerin localizes to the nucleolus (scale bar $=5$ $\mu \mathrm{m})$. 
A

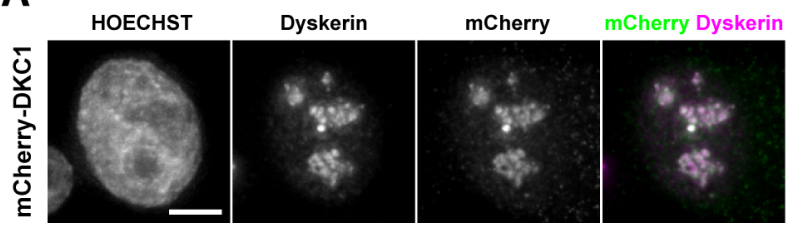

B $T R$
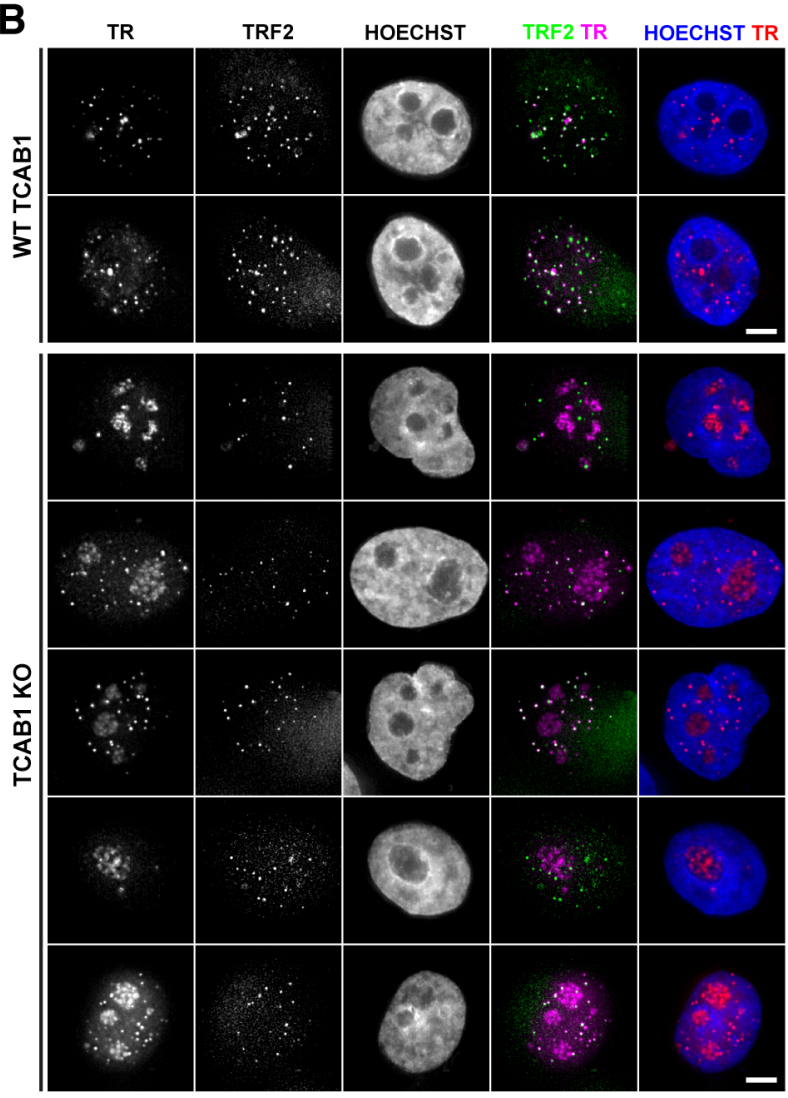

C

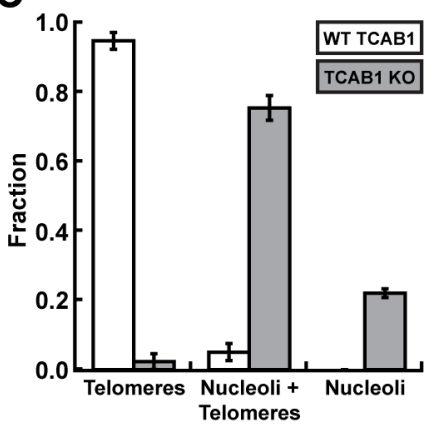

D

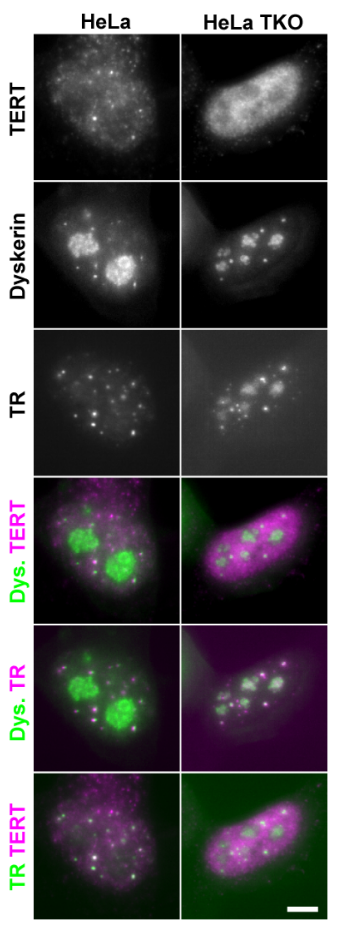

885

886

887

888

889

890

891

892

893

894

895

Figure 3 - Figure Supplement 1. (A) Images of HeLa cells transiently expressing mCherry-dyskerin probed with antibodies agains mCherry and dyskerin, demonstrating that mCherry dyskerin localizes to the nucleolus (scale bar $=5 \mu \mathrm{m}$ ). (B) Images of control and TCAB1 knock-out HeLa cells (3xFLAG-Halo-TERT, mEOS3.2-TRF2) overexpressing untagged TERT and TR. TR was detected using FISH and TRF2 was visualized using the fluorescence signal from mEOS3.2-TRF2. In control cells TR co-localizes with telomeres, while it is enriched in nucleoli and localized to telomeres in TCAB1 KO cells (scale bar $=5 \mu \mathrm{m}$ ). (C) Quantification of the fraction of cells showing TR localization exclusively to telomeres, to telomeres and nucleoli, or only to nucleoli ( 2 independent experiments, $>100$ cells per experiments, mean \pm standard deviation). (D) Images of HeLa cells and TCAB1 knockout cells transiently expressing untagged TERT and TR, probed with TERT (Abcam) and dyskerin antibodies and FISH for TR demonstrating that untagged TERT is excluded from nucleoli marked by dyskerin in parental and TCAB1 knock-out cells. 
bioRxiv preprint doi: https://doi org/10.1101/2021.05.27.445986; this version posted August 26, 2021. The copyright holder for this preprint (which was not certified by peer review) is the author/funder, who has granted bioRxiv a license to display the preprint in perpetuity. It is made available under aCC-BY-NC-ND 4.0 International license.

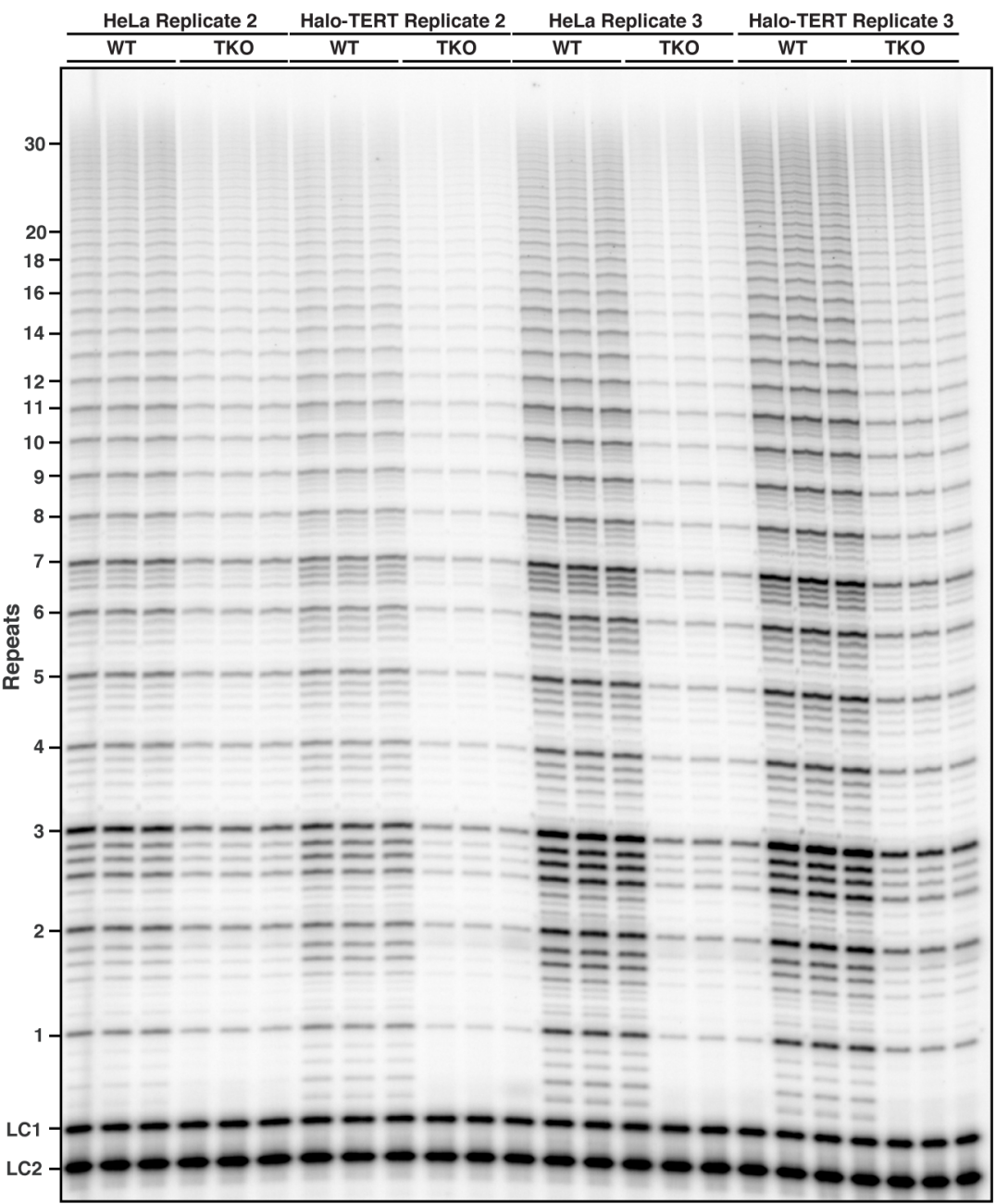

Figure 5 - Figure Supplement 1. Direct telomerase extension assay of telomerase immuno-purified from parental (WT) and TCAB1 knock-out (TKO) HeLa and Halo-TERT cell lines. LC1 and LC2, radiolabeled 900 
bioRxiv preprint doi: https://doi.org/10.1101/2021.05.27.445986; this version posted August 26, 2021. The copyright holder for this preprint

(which was not certified by peer review) is the author/funder, who has granted bioRxiv a license to display the preprint in perpetuity. It is made available under aCC-BY-NC-ND 4.0 International license.
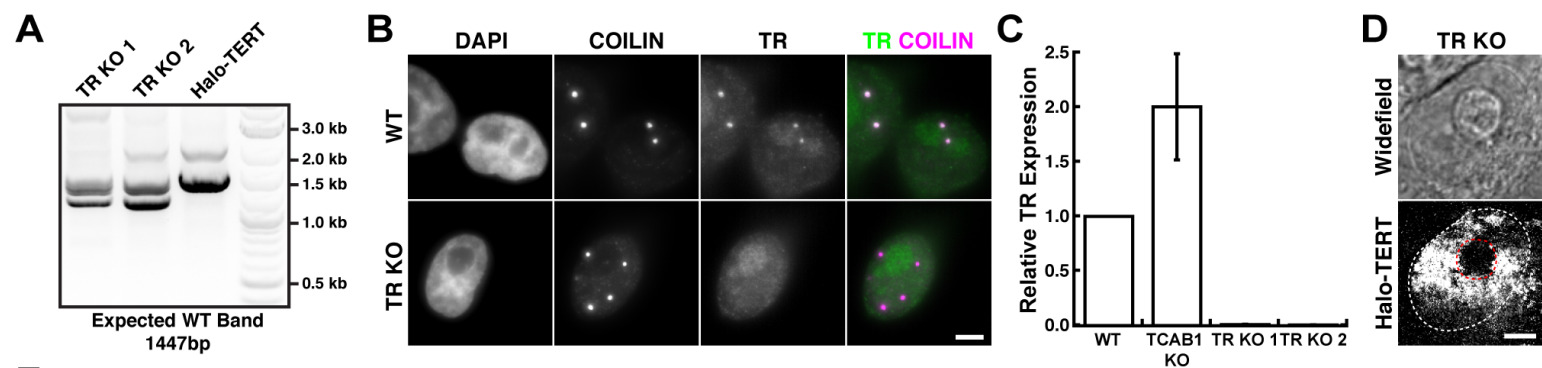

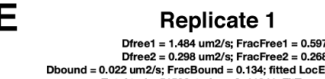
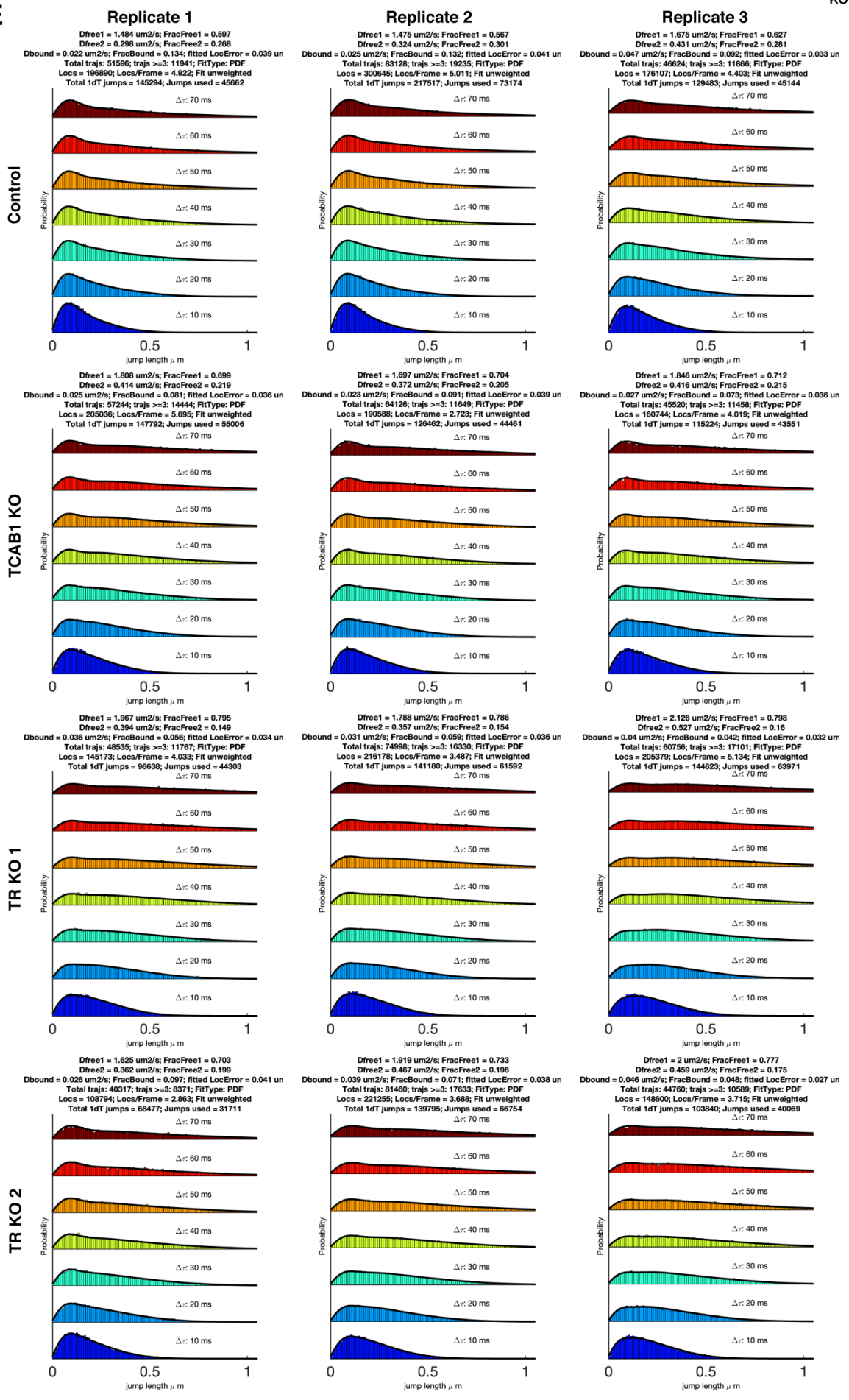
Figure 6 - Figure Supplement 1. (A) PCR analysis of the TR locus in parental and TR knock-out clones. Both TR knock-out clones show PCR products with reduced length that were confirmed to be knock-outs by Sanger sequencing. (B) Images of control and TR knock-out cells probed with an antibody against coilin and FISH probes specific for TR, demonstrating the lack of TR signal in TR knock-out cells (scale bar $=5 \mu \mathrm{m}$ ). (C) Determination of TR levels in control, TCAB1 knock-out, and TR knock-out cells, using RT-qPCR with primers specific to TR normalized to GAPDH (3 independent biological replicates, 3 technical replicates for each biological replicate, mean \pm standard deviation). (D) Maximum intensity projection of 2000 frames of a 3xFLAG-HaloTag (JF646) TERT movie (bottom), demonstrating that the TERT signal does not overlap with the nucleolus detected as circular shape in the transmitted light image (top, red dashed line). (E) Fitting of single-particle tracking data of TERT from control, TCAB1 knock-out, and TR knock-out cells expressing 3xFLAG-HaloTag-TERT using the Spot-On tool. 
bioRxiv preprint doi: https://doi.org/10 1101/2021.05.27.445986: this version posted August 26, 2021. The copyright holder for this preprint (which was not certified by peer review) is the author/funder, who has granted bioRxiv a license to display the preprint in perpetuity. It is made available under aCC-BY-NC-ND 4.0 International license.

A

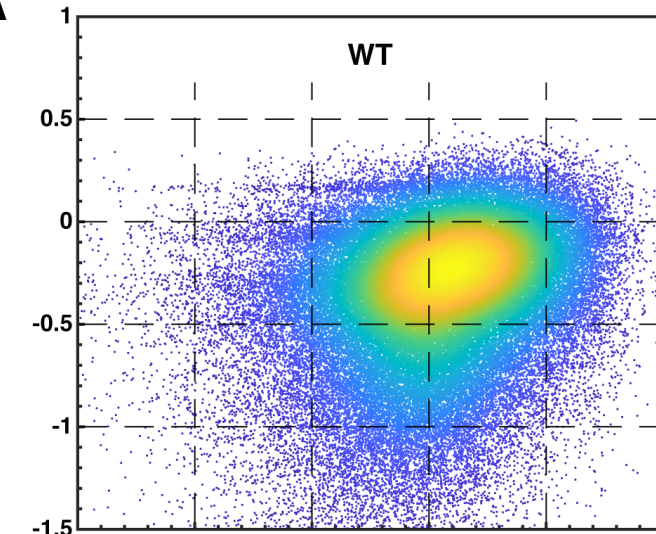

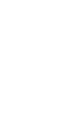

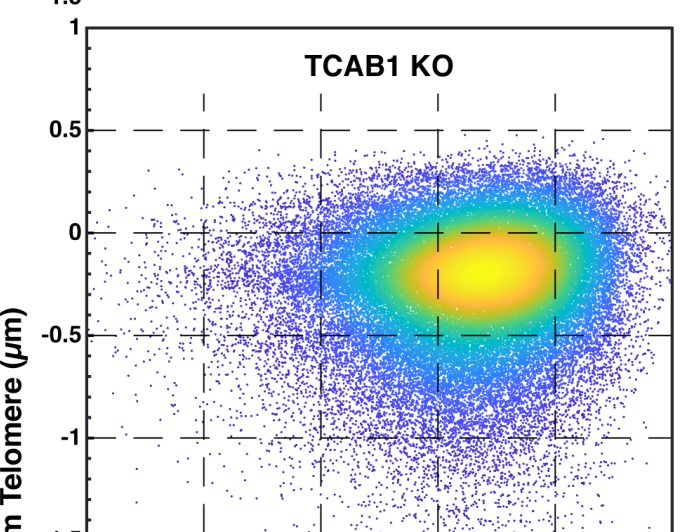

5
0
0
0
0
0
0
0
0

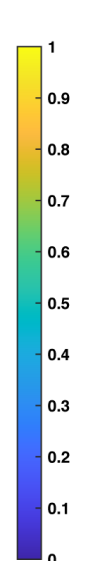

B

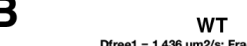

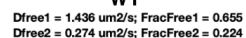

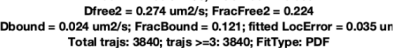

Locs $=$ 83546; Locs $/$ Frame $=0.659$; Fit unweighted

Tom

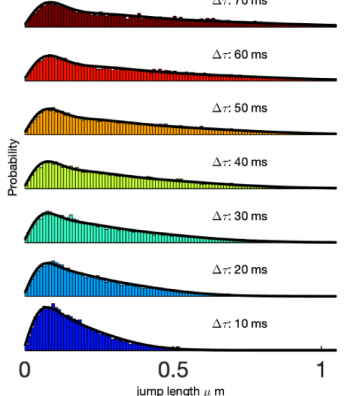

TCAB1 KO

Diree1 $=1.778 \mathrm{um} 2 / \mathrm{s} ; \mathrm{FracFree} 1=0.802$
Dfree2 $2=0.308 \mathrm{um} 2 / \mathrm{s} ; \mathrm{FracFree2}=0.156$

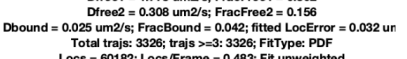

Total trajs: 3326 ; trajs $>3: 33326 ;$ Fiftype: PDF
Locs $=60182 ;$ Locs/Frame $=0.483$; Fit unweighted

Total 1dT jumps = 56856; Jumps used $=13304$

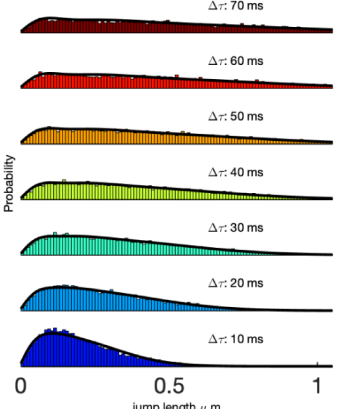

Jump length $\mu$

Dfree $1=20.58 \mathrm{um} 2 / \mathrm{s} ;$ FracFree $1=0.812$
Dtree $2=0.468 \mathrm{um} 2 / \mathrm{s} ;$ FracFree $2=0.144$

S 0.029 ur

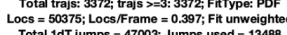

Total 1 d T jumps $=47003 ;$ Jumps used $=13488$

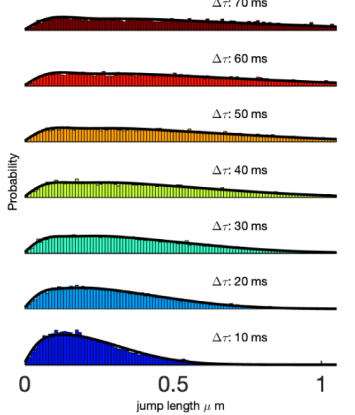

TR KO Clone 2

Diree $1=1.784$ un $2 / \mathrm{s}$; FracFree $1=0.815$
Dfree $2=0.342$ um 2 s: FracFree $2=0.135$

Dbound $=0.055$ um $2 / \mathrm{ss}_{\mathrm{s}}$ FracB Bund $=0.05$; fitted LocError $=0.032 \mathrm{ur}$

Locs $=36570 ;$ L Locs/Frame $=0.297$; Fit unwwioh

Lotal
Totat I jumps $=34025$; Jumps used $=10180$

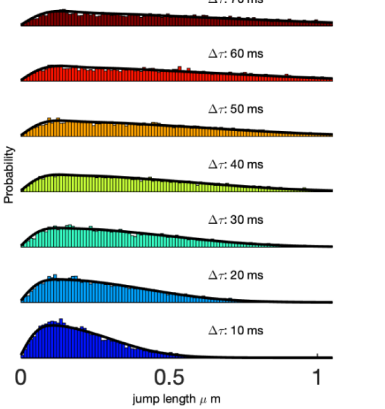


Figure 6 - Figure Supplement 2. (A) Analysis if the step size of telomeric TERT particles relative to the distance of the particle to the closest telomere (pooled results from 3 independent biological replicates with 19-30 cells analyzed per replicate). TERT molecules bound to the telomere are expected to have small step sizes and a short distance to the closest telomere, which is apparent in the enrichment of events in the lower quadrants in the WT control. This enrichment is not observed in TCAB1 and TR knock-out cells. (B) Spot-On analysis of telomeric TERT particles (pooled results from 3 independent biological replicates with 19-30 cells analyzed per replicate). The fraction of bound TERT particles in TCAB1 and TR knock-out cells is $4-5 \%$, compared to $12 \%$ in the WT control cells.

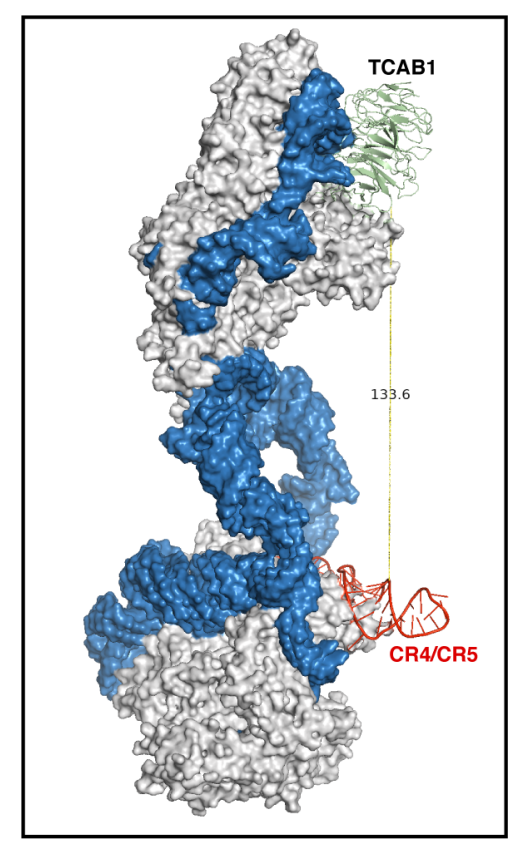

930

Figure 6 - Figure Supplement 3. (A) Structure of the telomerase RNP showing the distance between TCAB1 and CR4/CR5 (13.4 nm). TR in blue, CR4/CR5 in red, and TCAB1 in green (Ghanim et al., 2021). 


\section{Movie Legends}

933

934

935

936

937
Movie 1. Single-particle tracking of 3xFLAG-HaloTag-TERT labeled with JF646 in a control cell acquired at 100 frames per second. Trajectories with a minimum of 5 localizations are displayed. 150x150 pixels with a pixel size of $0.16 \mu \mathrm{m}$.

Movie 2. Single-particle tracking of 3xFLAG-HaloTag-TERT labeled with JF646 in a TCAB1 knockout cell acquired at 100 frames per second. Trajectories with a minimum of 5 localizations are displayed. $150 \times 150$ pixels with a pixel size of $0.16 \mu \mathrm{m}$.

Movie 3. Movie of cell expressing GFP-nucleolin (red) and 3xFLAG-HaloTag-NLS (green) labeled with JF646 acquired at 100 frames per second, showing overlap of 3xFLAGHaloTag-NLS with nucleoli. 140x140 pixels with a pixel size of $0.16 \mu \mathrm{m}$.

Movie 4. Movie of 3xFLAG-HaloTag-TERT labeled with JF646 in a control (left), TCAB1 knockout (middle), and TR knockout (right) cell acquired at 100 frames per second. Each panel is $150 \times 150$ pixels in size with a pixel size of $0.16 \mu \mathrm{m}$.

Movie 5. Single-particle tracking of 3xFLAG-HaloTag-TERT labeled with JF646 in a TR knockout cells acquired at 100 frames per second. Trajectories with a minimum of 5 localizations are displayed. $150 \times 150$ pixels with a pixel size of $0.16 \mu \mathrm{m}$. 